Article

\title{
Alcohol Participates in the Synthesis of Functionalized Coumarin-Fused Pyrazolo[3,4-b]Pyridine from a One-Pot Three-Component Reaction
}

\author{
Wei Lin ${ }^{1, *}{ }^{\mathbb{C}}$, Cangwei Zhuang ${ }^{1}$, Xiuxiu $\mathrm{Hu}^{1}{ }^{1}$, Juanjuan Zhang ${ }^{2}$ and Juxian Wang ${ }^{3, *}$ \\ 1 School of Chemistry and Environmental Engineering, Jiangsu University of Technology, \\ Changzhou 213001, China \\ 2 State Key Laboratory of Pharmaceutical Biotechnology, Nanjing University, Nanjing 210093, China \\ 3 Institute of Medicinal Biotechnology, Chinese Academy of Medical Science and Peking Union Medical \\ College, Beijing 100050, China \\ * Correspondence: linwei@jsut.edu.cn (W.L.); imbjxwang@163.com (J.-W.) \\ Academic Editors: Derek J. McPhee and Roman Dembinski \\ Received: 10 July 2019; Accepted: 2 August 2019; Published: 4 August 2019

\begin{abstract}
A concise and efficient approach to synthesizing coumarin-fused pyrazolo[3,4-b]pyridine via silica sulfuric acid (SSA) catalyzed three-component domino reaction under microwave irradiation has been demonstrated. Participation of various alcohols in construction of coumarin derivatives has been described for the first time. Short reaction time, high yields, one-pot procedure, usage of eco-friendly catalyst, and solvent are the key features of this method.
\end{abstract}

Keywords: coumarin; pyrazolo[3,4-b]pyridine; synthesis; silica sulfuric acid

\section{Introduction}

As one of the most important heterocyclic compounds, coumarin was widely found in nature products [1,2], and several synthetic coumarins [3] with a variety of pharmacophoric groups at C-3, C-4, and C-7 positions have been intensively screened for various biological activities like AChE inhibitors [4-6], anticancer [7-9], anticoagulant [10,11], anti-HIV [12-14], antitubercular [15,16], anti-inflammatory [17,18], antioxidant [19], antibacterial [20], antihypertensive [21], anticonvulsant [22], antifungal [23], and antihyperglycemic [24]. A recent literature survey suggests quite a few coumarin derivatives have been patented for their biological properties (Figure 1). Besides the high biological activity, coumarin is also considered to be a functional material $[25,26]$ such as receptors [27-29], signaling units in sensors and biosensors, as well as in advanced photophysical systems [30,31].

Among various nitrogen-containing heterocyclic compounds, pyrazolo[3,4-b]pyridine is recognized as important drug molecular skeleton in recent years due to a wide varieties of biological activities (Figure 2), such as antimicrobial [32,33], anti-inflammatory [34,35], anti-proliferative [36,37], and many other $[38,39]$ important effects. 

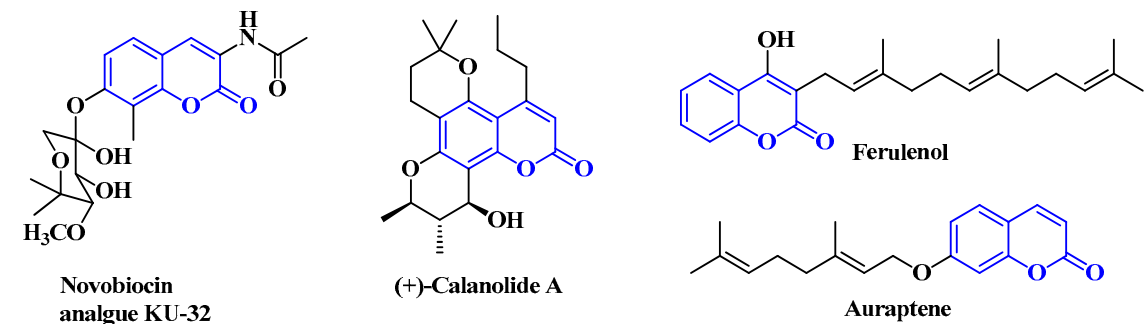

analgue $\mathrm{KU}-32$

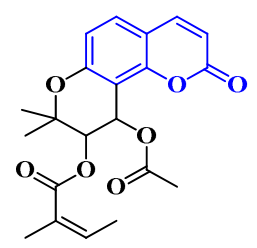

Praeruptorin A

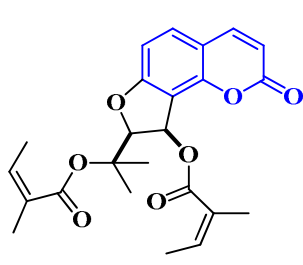

Archangelicin

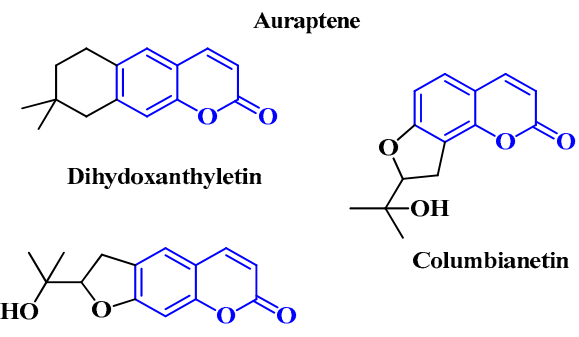

Marmesin

Figure 1. General structures of coumarin molecules possessing biological activity.

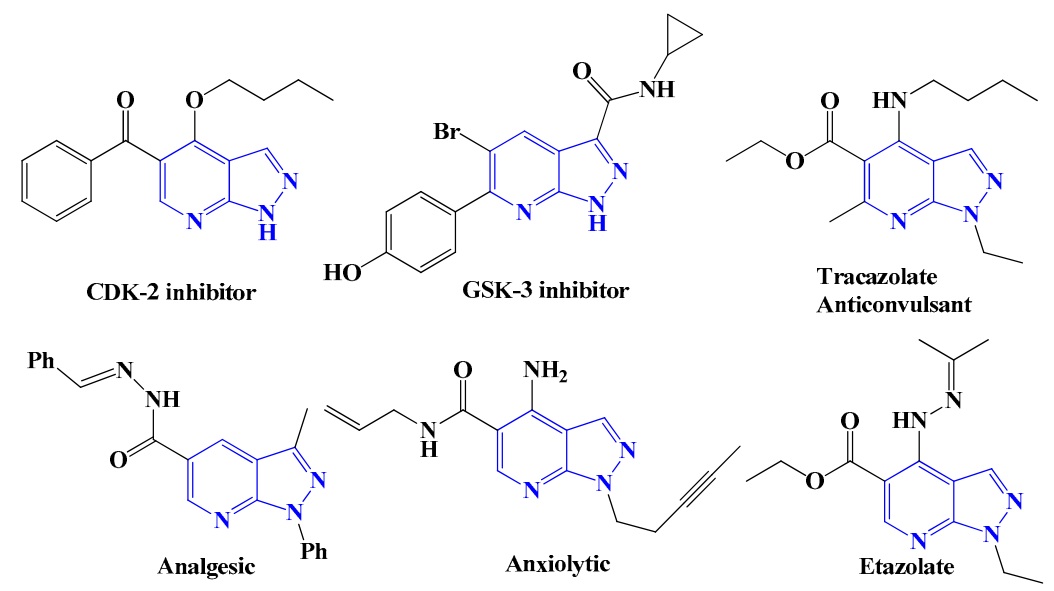

Figure 2. Biologically active compounds having pyrazolo[3,4-b]pyridine unit.

Therefore, development and introduction of a convenient, efficient method for the synthesis of coumarin-fused pyrazolo[3,4-b]pyridine is highly desirable for their immense pharmacological potential. As a part of our research on the synthesis of novel functionalized heterocyclic derivatives [40-46], in the current paper, we report a novel three-component domino reaction for the synthesis of functionalized coumarin-fused pyrazolo[3,4-b]pyridine derivatives using silica sulfuric acid as the catalyst. It worth mentioning that participation of alcohols in construction of coumarin derivatives is described for the first time.

\section{Results and Discussion}

In the early literature reports of our group [44], the coumarino[4,3-d]pyrazolo[3,4-b]pyridine derivative (3a) was synthesized by the reaction of 3-acylcoumarin (1a) with 5-aminopyrazole (2a) catalyzed by silica sulfuric acid (SSA) in $\mathrm{EtOH}$ at $90{ }^{\circ} \mathrm{C}$ for 20 min under microwave irradiation (Scheme 1). 


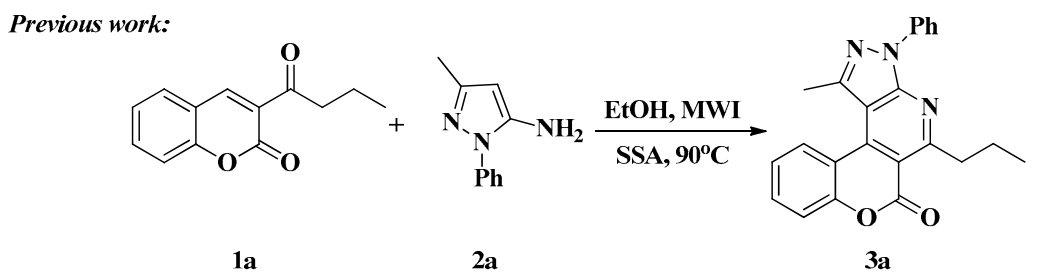

Scheme 1. Synthesis of coumarino[4,3-d]pyrazolo[3,4-b]pyridine derivative 3a.

According to our previously reported synthetic procedure, we speculate that the coumarin derivative $\mathbf{6 a}$ could be obtained from the 2-butyryl-3H-benzo[f $]$ chromen-3-one (4a) and 3-methyl-1phenyl-1H-pyrazol-5-amine (2a) used as the starting materials. However, product $\mathbf{6 a}$ was not available as expect (Scheme 2-1). Considering the steric hindrance effect of the reaction, when ethanol and ethylene glycol $(\mathrm{EG})$ as mixed solvent (volume ratio of $\mathrm{EG} / \mathrm{EtOH}=1: 1$ ) was added to the reaction, and further increasing the temperature $\left(120^{\circ} \mathrm{C}\right)$, a new product $7 \mathbf{a}$ formed unexpectedly (Scheme 2-2), which was identified by ${ }^{1} \mathrm{H}-\mathrm{NMR},{ }^{13} \mathrm{C}-\mathrm{NMR}$, HRMS analysis. Moreover, we also obtained the single crystal of 7a suitable for X-ray analysis (Figure 3) [47]. To our surprise, the solvent ethanol also participated in this reaction and a novel coumarin derivative was constructed.

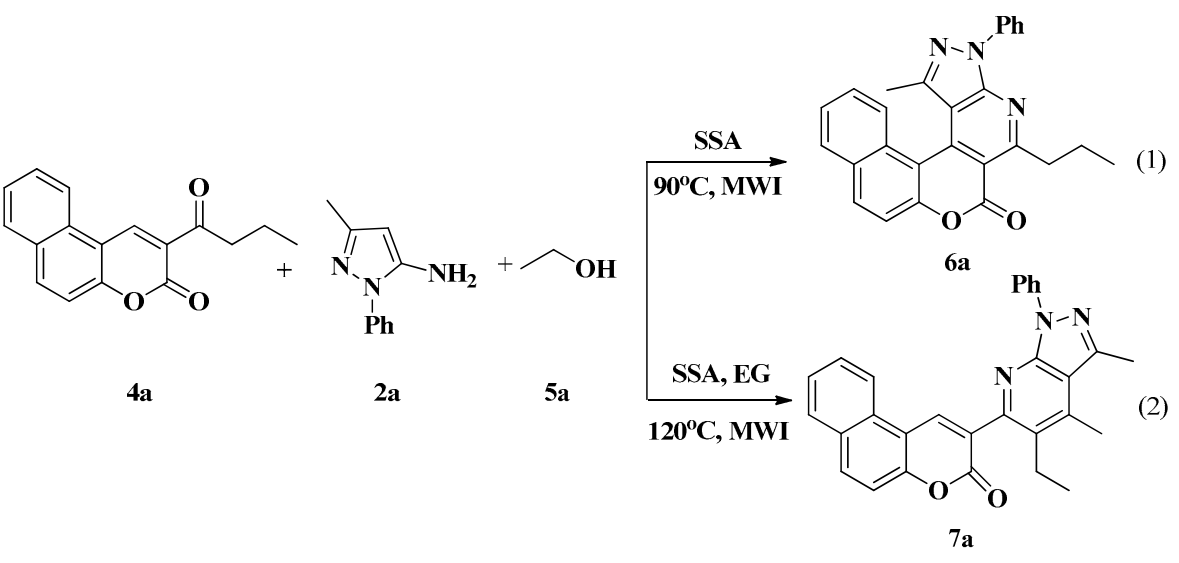

Scheme 2. New multicomponent domino reactions.

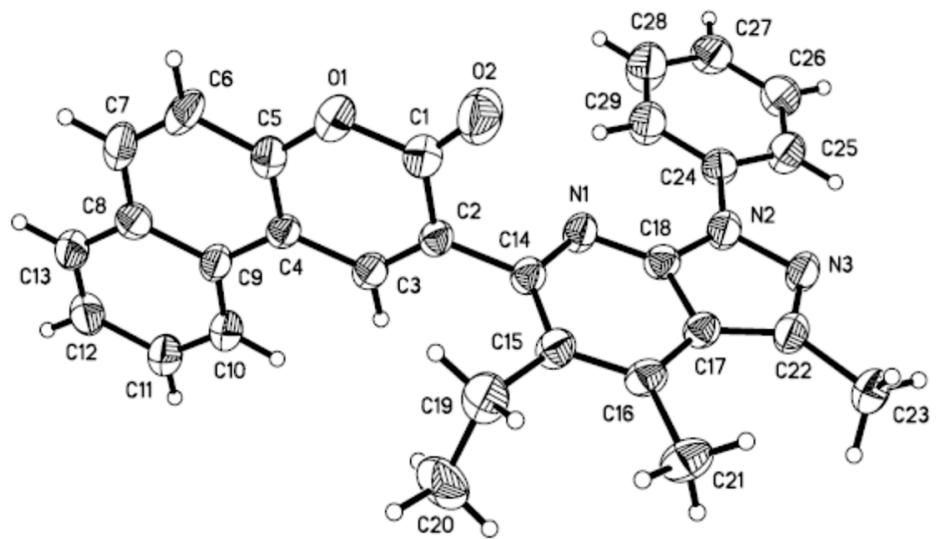

Figure 3. Crystal structure of 7a.

In order to achieve the optimal conditions of three-component reaction, a series of catalysts, solvents, and temperature were screened, as shown in Table 1 . Some other acid catalysts such as $p$ - TsOH, $\mathrm{HClO}_{3} \mathrm{~S}, \mathrm{H}_{2} \mathrm{SO}_{4}, \mathrm{SiO}_{2}-\mathrm{H}_{2} \mathrm{SO}_{4}$ (Table 1. entries 1, 3-5) and base catalysts such as $\mathrm{K}_{2} \mathrm{CO}_{3}$, $\mathrm{NaOH}, \mathrm{Cs}_{2} \mathrm{CO}_{3}$ (Table 1, entries 6-8) were tested. However, none of them gave better results, lead to the identification of SSA as the most effective catalyst (Table 1. entry 2). To further increase the yield 
of desired product 7a, different solvents were evaluated. The results revealed that EtOH and EG as mixed solvents greatly improved the transformation, in control to EtOH, PEG, glycerol, and DMF as a single solvent (Table 1, entries 2, 9-12). When the volume ratio of EG/EtOH = 3:1, the yield of 7a could further increase to $68 \%$ (Table 1, entry 15). Much to our delight, we observed that increasing of the temperature to $140{ }^{\circ} \mathrm{C}$ resulted in affording $7 \mathbf{a}$ in $84 \%$ yield (Table 1 , entry 20 ).

Table 1. Optimizing the reaction conditions for the synthesis of $7 \mathrm{a}$ under microwave ${ }^{\mathrm{a}}$.

\begin{tabular}{|c|c|c|c|c|}
\hline Entry & Catalyst & Solvent $(v / v)$ & Temperature $\left({ }^{\circ} \mathrm{C}\right)$ & Yield (\%) ${ }^{b}$ \\
\hline 1 & $p$-TsOH (20 mol\%) & $\mathrm{EG} / \mathrm{EtOH}=1: 1$ & 120 & trace \\
\hline 2 & SSA $(0.25 \mathrm{~g})$ & $\mathrm{EG} / \mathrm{EtOH}=1: 1$ & 120 & 58 \\
\hline 3 & $\mathrm{HClO}_{3} \mathrm{~S}(5 \mathrm{~mol} \%)$ & $\mathrm{EG} / \mathrm{EtOH}=1: 1$ & 120 & 36 \\
\hline 4 & $\mathrm{SiO}_{2}-\mathrm{H}_{2} \mathrm{SO}_{4}(0.25 \mathrm{~g})$ & $\mathrm{EG} / \mathrm{EtOH}=1: 1$ & 120 & - \\
\hline 5 & $\mathrm{H}_{2} \mathrm{SO}_{4}(20 \mathrm{~mol} \%)$ & $\mathrm{EG} / \mathrm{EtOH}=1: 1$ & 120 & - \\
\hline 6 & $\mathrm{~K}_{2} \mathrm{CO}_{3}(20 \mathrm{~mol} \%)$ & $\mathrm{EG} / \mathrm{EtOH}=1: 1$ & 120 & - \\
\hline 7 & $\mathrm{NaOH}(20 \mathrm{~mol} \%)$ & $\mathrm{EG} / \mathrm{EtOH}=1: 1$ & 120 & - \\
\hline 8 & $\mathrm{Cs}_{2} \mathrm{CO}_{3}(20 \mathrm{~mol} \%)$ & $\mathrm{EG} / \mathrm{EtOH}=1: 1$ & 120 & - \\
\hline 9 & SSA $(0.25 \mathrm{~g})$ & $\mathrm{EtOH}$ & 110 & 20 \\
\hline 10 & SSA (0.25 g) & PEG/EtOH = 1:1 & 120 & 45 \\
\hline 11 & SSA $(0.25 \mathrm{~g})$ & Glycerol/EtOH = 1:1 & 120 & 32 \\
\hline 12 & SSA (0.25 g) & $\mathrm{DMF} / \mathrm{EtOH}=1: 1$ & 120 & 24 \\
\hline 13 & SSA (0.25 g) & $\mathrm{EG} / \mathrm{EtOH}=1: 2$ & 120 & 21 \\
\hline 14 & SSA (0.25 g) & $\mathrm{EG} / \mathrm{EtOH}=2: 1$ & 120 & 55 \\
\hline 15 & SSA (0.25 g) & $\mathrm{EG} / \mathrm{EtOH}=3: 1$ & 120 & 68 \\
\hline 16 & SSA (0.25 g) & $\mathrm{EG} / \mathrm{EtOH}=4: 1$ & 120 & 57 \\
\hline 17 & SSA (0.25 g) & $\mathrm{EG} / \mathrm{EtOH}=3: 1$ & 100 & trace \\
\hline 18 & SSA (0.25 g) & $\mathrm{EG} / \mathrm{EtOH}=3: 1$ & 110 & trace \\
\hline 19 & SSA (0.25 g) & $\mathrm{EG} / \mathrm{EtOH}=3: 1$ & 130 & 78 \\
\hline 20 & SSA (0.25 g) & $\mathrm{EG} / \mathrm{EtOH}=3: 1$ & 140 & 84 \\
\hline 21 & SSA (0.25 g) & $\mathrm{EG} / \mathrm{EtOH}=3: 1$ & 150 & 76 \\
\hline
\end{tabular}

a Reaction conditions: $\mathbf{4 a}(0.5 \mathrm{mmol}), \mathbf{2 a}(0.5 \mathrm{mmol}), \mathbf{5 a}(1.0 \mathrm{~mL}), 45 \mathrm{~min} ;{ }^{\mathrm{b}} \mathrm{GC}$ yield of $\mathbf{7 a}$ determined using tridecane as internal standard.

With optimal conditions in hand, the corresponding novel coumarin-fused pyrazolo[3,4- $b]$ pyridine derivatives 7 were synthesized (Scheme 3).

As illustrated in Scheme 3, the substrate scope of the transformation was examined using arylbenzo[f]chromen-3-one 4, enaminone 2, and alkyl alcohol 5 as staring materials. Notably, electronic effects had an important impact on this reaction. When the substituent $\mathrm{R}^{3}$ was electron-donating group, such as $\mathrm{Me}$, the desired products could not be obtained at all $(\mathbf{7 e}, \mathbf{7 f})$.

To further expand the scope of substrates, aryl alcohols (8) instead of alkyl alcohols (5) were also tested. It was found that aryl alcohols were well tolerated under the optimal reaction conditions, the corresponding products were afforded in moderate to good yields. When substituent $\mathrm{R}^{3}$ was electron-withdrawing groups (Ph), the yields were good and no more than $1 \mathrm{~h}$ cost (Table 2, entries 1-19). However, the substituents $\mathrm{R}^{3}$ was electron-donating groups $\left(\mathrm{CH}_{3}\right)$ (Table 2, entries 20-22), the yields were lower and the reaction time was longer. Unfortunately, When $\mathrm{R}^{3}$ and $\mathrm{R}^{4}$ was electron rich group, such as Me, the reaction could not proceed successfully (Table 2, entry 23). 
Table 2. Synthesis of coumarin-fused pyrazolo[3,4-b]pyridine derivatives $9^{\mathrm{a}}$.

\begin{tabular}{|c|c|c|c|c|c|c|c|c|}
\hline & & & 2 & & 8 & & 9 & \\
\hline Entry & Product & $\mathbf{R}^{1}$ & $\mathbf{R}^{2}$ & $\mathbf{R}^{3}$ & $\mathbf{R}^{4}$ & Ar & Time (h) & Isolated yield $(\%)$ \\
\hline 1 & $9 a$ & $\mathrm{CH}_{2} \mathrm{CH}_{3}$ & $\mathrm{H}$ & $\mathrm{Ph}$ & $\mathrm{CH}_{3}$ & $\mathrm{C}_{6} \mathrm{H}_{5}$ & 1 & 69 \\
\hline 2 & $9 b$ & $\mathrm{CH}_{2} \mathrm{CH}_{3}$ & $\mathrm{H}$ & $\mathrm{Ph}$ & $\mathrm{CH}_{3}$ & $4-\mathrm{CH}_{3} \mathrm{C}_{6} \mathrm{H}_{4}$ & 1 & 74 \\
\hline 3 & 9c & $\mathrm{CH}_{2} \mathrm{CH}_{3}$ & $\mathrm{H}$ & $\mathrm{Ph}$ & $\mathrm{CH}_{3}$ & $4-\mathrm{OCH}_{3} \mathrm{C}_{6} \mathrm{H}_{4}$ & 0.75 & 77 \\
\hline 4 & $9 d$ & $\mathrm{CH}_{2} \mathrm{CH}_{3}$ & $\mathrm{H}$ & $\mathrm{Ph}$ & $\mathrm{CH}_{3}$ & $3-\mathrm{OCH}_{3} \mathrm{C}_{6} \mathrm{H}_{4}$ & 0.75 & 76 \\
\hline 5 & $9 e$ & $\mathrm{CH}_{2} \mathrm{CH}_{3}$ & $\mathrm{H}$ & $\mathrm{Ph}$ & $\mathrm{CH}_{3}$ & $4-\mathrm{BrC}_{6} \mathrm{H}_{4}$ & 0.75 & 71 \\
\hline 6 & $9 f$ & $\mathrm{CH}_{2} \mathrm{CH}_{3}$ & $\mathrm{H}$ & $\mathrm{Ph}$ & $\mathrm{CH}_{3}$ & Pyridine-4-yl & 1 & 70 \\
\hline 7 & $9 g$ & $\mathrm{CH}_{2} \mathrm{CH}_{3}$ & $\mathrm{H}$ & $\mathrm{Ph}$ & $\mathrm{CH}_{3}$ & Furan-2-yl & 1 & 76 \\
\hline 8 & $9 \mathrm{~h}$ & $\mathrm{CH}_{2} \mathrm{CH}_{3}$ & $\mathrm{OCH}_{3}$ & $\mathrm{Ph}$ & $\mathrm{CH}_{3}$ & $\mathrm{C}_{6} \mathrm{H}_{5}$ & 1 & 62 \\
\hline 9 & $9 \mathrm{i}$ & $\mathrm{CH}_{2} \mathrm{CH}_{3}$ & $\mathrm{OCH}_{3}$ & $\mathrm{Ph}$ & $\mathrm{CH}_{3}$ & $4-\mathrm{CH}_{3} \mathrm{C}_{6} \mathrm{H}_{4}$ & 1 & 70 \\
\hline 10 & $9 j$ & $\mathrm{CH}_{2} \mathrm{CH}_{3}$ & $\mathrm{OCH}_{3}$ & $\mathrm{Ph}$ & $\mathrm{CH}_{3}$ & $4-\mathrm{OCH}_{3} \mathrm{C}_{6} \mathrm{H}_{4}$ & 1 & 72 \\
\hline 11 & 9k & $\mathrm{CH}_{3}$ & $\mathrm{H}$ & $\mathrm{Ph}$ & $\mathrm{CH}_{3}$ & $\mathrm{C}_{6} \mathrm{H}_{5}$ & 1 & 68 \\
\hline 12 & 91 & $\mathrm{CH}_{3}$ & $\mathrm{H}$ & $\mathrm{Ph}$ & $\mathrm{CH}_{3}$ & $4-\mathrm{CH}_{3} \mathrm{C}_{6} \mathrm{H}_{4}$ & 1.25 & 70 \\
\hline 13 & $9 \mathrm{~m}$ & $\mathrm{CH}_{3}$ & $\mathrm{H}$ & $\mathrm{Ph}$ & $\mathrm{CH}_{3}$ & $4-\mathrm{OCH}_{3} \mathrm{C}_{6} \mathrm{H}_{4}$ & 1.25 & 74 \\
\hline 14 & $9 n$ & $\mathrm{CH}_{3}$ & $\mathrm{H}$ & $\mathrm{Ph}$ & $\mathrm{CH}_{3}$ & $3-\mathrm{OCH}_{3} \mathrm{C}_{6} \mathrm{H}_{4}$ & 1.25 & 72 \\
\hline 15 & 9o & $\mathrm{CH}_{3}$ & $\mathrm{OCH}_{3}$ & $\mathrm{Ph}$ & $\mathrm{CH}_{3}$ & $4-\mathrm{CH}_{3} \mathrm{C}_{6} \mathrm{H}_{4}$ & 1.25 & 67 \\
\hline 16 & $9 p$ & $\mathrm{CH}_{3}$ & $\mathrm{OCH}_{3}$ & $\mathrm{Ph}$ & $\mathrm{CH}_{3}$ & $4-\mathrm{OCH}_{3} \mathrm{C}_{6} \mathrm{H}_{4}$ & 1.25 & 70 \\
\hline 17 & $9 q$ & $\mathrm{CH}_{3}$ & $\mathrm{OCH}_{3}$ & $\mathrm{Ph}$ & $\mathrm{CH}_{3}$ & $\mathrm{C}_{6} \mathrm{H}_{5}$ & 1.25 & 60 \\
\hline 18 & $9 \mathrm{r}$ & $\mathrm{H}$ & $\mathrm{H}$ & $\mathrm{Ph}$ & $\mathrm{CH}_{3}$ & $\mathrm{C}_{6} \mathrm{H}_{5}$ & 1.5 & 56 \\
\hline 19 & $9 \mathrm{~s}$ & $\mathrm{H}$ & $\mathrm{H}$ & $\mathrm{Ph}$ & $\mathrm{CH}_{3}$ & $4-\mathrm{OCH}_{3} \mathrm{C}_{6} \mathrm{H}_{4}$ & 1.5 & 58 \\
\hline 20 & $9 t$ & $\mathrm{CH}_{2} \mathrm{CH}_{3}$ & $\mathrm{H}$ & $\mathrm{CH}_{3}$ & $\mathrm{Ph}$ & $\mathrm{C}_{6} \mathrm{H}_{5}$ & 2 & 55 \\
\hline 21 & $9 u$ & $\mathrm{CH}_{3}$ & $\mathrm{OCH}_{3}$ & $\mathrm{CH}_{3}$ & $\mathrm{Ph}$ & $\mathrm{C}_{6} \mathrm{H}_{5}$ & 2 & 50 \\
\hline 22 & $9 v$ & $\mathrm{CH}_{3}$ & $\mathrm{OCH}_{3}$ & $\mathrm{CH}_{3}$ & $\mathrm{Ph}$ & $4-\mathrm{OCH}_{3} \mathrm{C}_{6} \mathrm{H}_{4}$ & 2 & 45 \\
\hline 23 & $9 w$ & $\mathrm{CH}_{2} \mathrm{CH}_{3}$ & $\mathrm{H}$ & $\mathrm{CH}_{3}$ & $\mathrm{CH}_{3}$ & $4-\mathrm{OCH}_{3} \mathrm{C}_{6} \mathrm{H}_{4}$ & 2.5 & trace \\
\hline
\end{tabular}

a Reaction conditions: arylbenzo[f $]$ chromen-3-one 4 ( $0.5 \mathrm{mmol})$, enaminone 2 (0.5 mmol), aryl alcohols $8(1.0 \mathrm{~mL})$, EG $(3 \mathrm{~mL})$ and SSA $(0.25 \mathrm{~g}), 140{ }^{\circ} \mathrm{C}$.

To gain insight into the mechanism of this one-spot three-component reaction process, some additional experiments were performed. When benzaldehyde (10) was added to the reaction instead of phenylmethanol (8a) under standard conditions, 73\% yield of desired product (9a) could be obtained, and reaction time reduced from $1 \mathrm{~h}$ to $15 \mathrm{~min}$ (Scheme $4 \mathrm{~A}$ ), and when butyraldehyde (11) was added to the reaction $50 \%$ yield of desired product $(7 \mathrm{c}$ ) could be obtained (Scheme $4 \mathrm{~B}$ ). The reaction did not proceed successfully without SSA catalyzed. Just phenylmethanol (8a) was heated to $140{ }^{\circ} \mathrm{C}$, directly with the catalyst of SSA, benzaldehyde (10) and benzoic acid (12) could be detected by GC-MS (Scheme 4C). We speculated that the benzaldehyde was most likely the key intermediate in this protocol. 


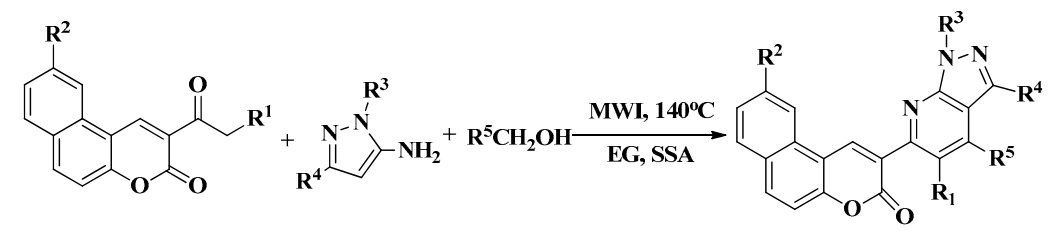<smiles>CCc1c(-c2cc3c(ccc4ccccc43)oc2=O)nc2c(c(C)nn2-c2ccccc2)c1C</smiles>

7a, Yield $84 \%{ }^{b}$<smiles>CCc1c(-c2cc3c(ccc4ccc(OC)cc43)oc2=O)nc2c(c(C)nn2-c2ccccc2)c1C</smiles>

7d Yield $84 \%{ }^{b}$<smiles></smiles>

7 b Yield $69 \%^{\mathrm{b}}$<smiles>CCc1c(-c2cc3c(ccc4ccccc43)oc2=O)nc2c(c(-c3ccccc3)nn2C)c1C</smiles>

7 e trace $^{c}$<smiles>CCCc1c(CC)c(-c2cc3c(ccc4ccccc43)oc2=O)nc2c1C(C)=N[C@H]2c1ccccc1</smiles>

$7 c$ Yield $64 \%$ b<smiles>CCc1c(-c2cc3c(ccc4ccccc43)oc2=O)nc2c(c(C)nn2C)c1C</smiles>

$7 f$ trace $^{c}$

Scheme 3. Synthesis of coumarin-fused pyrazolo[3,4- $b]$ pyridine derivatives $7^{a}$. a Reaction conditions: arylbenzo[f $]$ chromen-3-one $4(0.5 \mathrm{mmol})$, enaminone $2(0.5 \mathrm{mmol})$, alkyl alcohol $5(1.0 \mathrm{~mL}), \mathrm{EG}(3 \mathrm{~mL})$ and SSA $(0.25 \mathrm{~g}), 140{ }^{\circ} \mathrm{C}, 45 \mathrm{~min} ;{ }^{\mathrm{b}}$ Isolated yield; ${ }^{\mathrm{c}} 2 \mathrm{~h}$.

A)<smiles>CCCC(=O)c1cc2c(ccc3ccccc32)oc1=O</smiles>

$4 \mathbf{a}$<smiles>Cc1cc(N)n(-c2ccccc2)n1</smiles>

2a<smiles>O=Cc1ccccc1</smiles>

10<smiles>CCc1c(-c2cc3c(ccc4ccccc43)oc2=O)nc2c(c(C)nn2-c2ccccc2)c1-c1ccccc1</smiles>

9a, 15 min, $73 \%$

B)

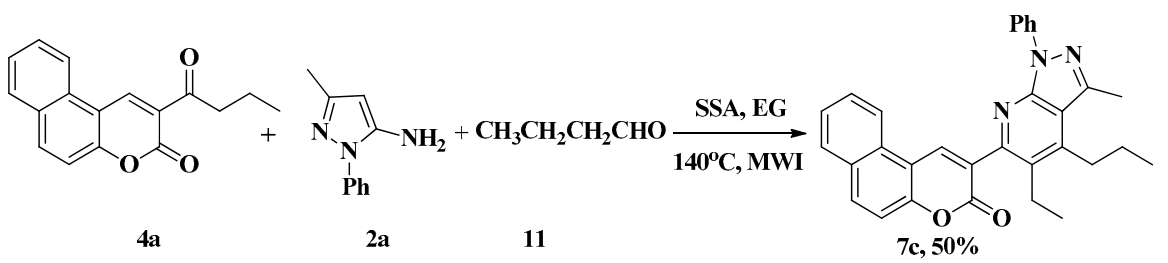

C)

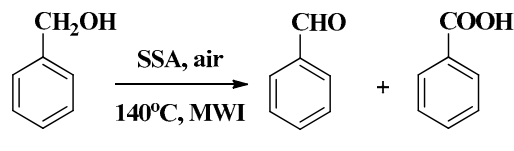

$8 \mathrm{a}$

10

12

Scheme 4. Preliminary mechanistic studies. (A) Synthesis of coumarin-fused pyrazolo[3,4-b]pyridine derivatives 9a. (B) Synthesis of coumarin-fused pyrazolo[3,4-b]pyridine derivatives 7c. (C) Reaction of phenylmethanol with the catalyst of SSA.

Herein, we propose the following mechanism for the reaction (Scheme 5). SSA catalyzed alkyl alcohol 5 to afford the corresponding aldehyde, then the intermediate $\mathbf{A}$ is formed by means of a 
Knoevenagel condensation of aldehyde and arylbenzo[f]chromen-3-one (4). The intermediate $\mathbf{A}$ is activated by SSA, which subsequently undergoes Michael addition with enaminone (2) via attack of the nucleophilic C-4 of the intermediate $\mathbf{A}$ to give intermediate $\mathbf{B}$, which transformed to more-stable intermediate $\mathbf{C}$. Then, intermediate $\mathbf{C}$ tautomerizes to intermediate $\mathbf{D}$, which undergoes intramolecular nucleophilic addition to form intermediate $\mathbf{E}$. In the last step, loss of $\mathrm{H}_{2} \mathrm{O}$ affords the desired product.

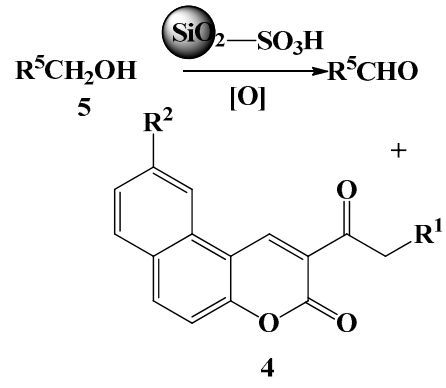

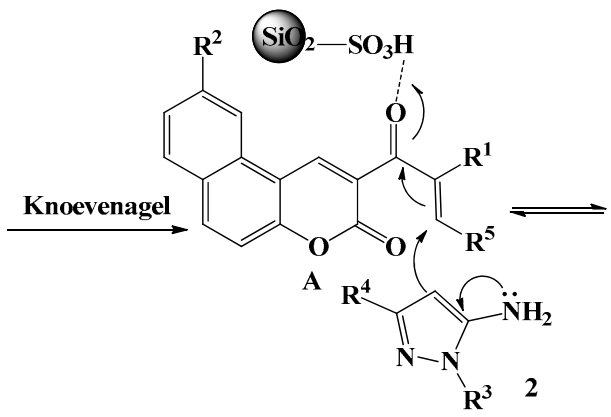

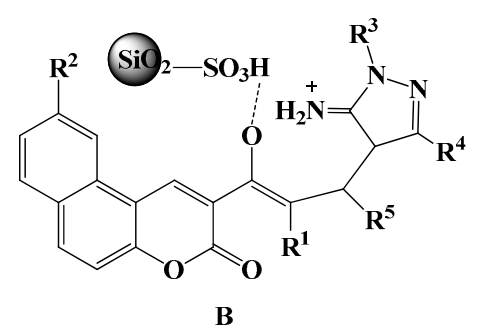

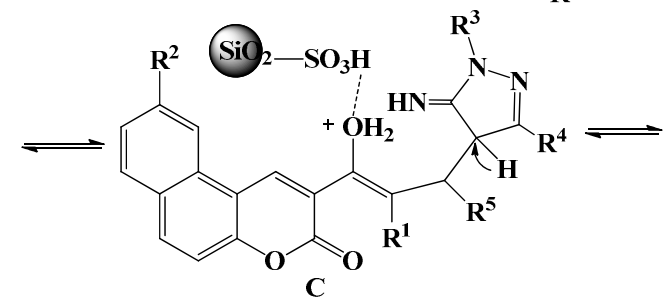

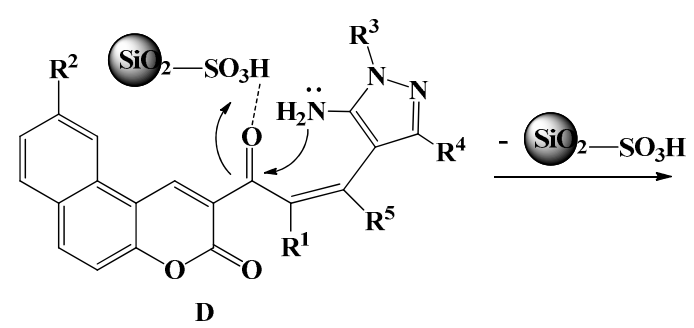

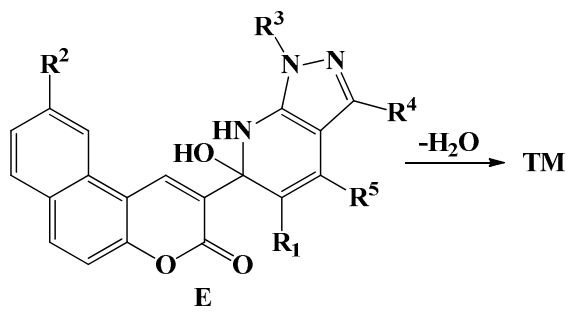

Scheme 5. Proposed mechanism for this reaction.

\section{Conclusions}

In conclusion, we have developed a protocol for the facile synthesis of various potentially biologically active coumarin-fused pyrazolo[3,4-b]pyridine derivatives, based on a novel threecomponent domino reaction under microwave irradiation. Using this method, coumarin derivatives could be rapidly constructed in moderate-to-good yields with short reaction time. Further study to deeply understand the reaction mechanism is currently underway in our lab.

\section{Experimental Section}

\subsection{General}

All reagents were purchased from commercial suppliers (Aladdin, Shanghai, China) and used without further purification. Microwave irradiation was carried out with Initiator 2.5 Microwave Synthesizers from Biotage, Uppsala, Sweden. The reaction temperatures were measured by infrared detector during microwave heating. Melting points are uncorrected. IR spectra were recorded on a Tensor 27 spectrometer (Bruker Corp., Karlsruhe, Germany) in KBr with absorptions in $\mathrm{cm}^{-1} .{ }^{1} \mathrm{H}-\mathrm{NMR}$ $(400 \mathrm{MHz})$ and ${ }^{13} \mathrm{C}-\mathrm{NMR}(75 \mathrm{MHz}$ or $100 \mathrm{MHz})$ spectra were recorded on a Varian Inova-400 $\mathrm{MHz}$ or Varian Inova-300 MHz (Varian, CA, America) in $\mathrm{CDCl}_{3}$, DMSO- $d_{6}$ or $\mathrm{CF}_{3} \mathrm{COOD}$ as solution. J values are in hertz. Chemical shifts are expressed in parts per million downfield from interal standard TMS. High-resolution mass spectra (HRMS) for all the compounds were determined on Bruker MicrOTOF-QII 
mass spectrometer (Bruker Corp., Karlsruhe, Germany) with ESI resource. X-ray diffraction analysis was recorded on a Smart-1000 diffractometer (PANalytical B.V., Holland).

\subsection{General Procedure for the Synthesis of Products 4 Are Represented as Follows}

Typically, 2-hydroxy-1-naphthaldehyde ( $5 \mathrm{mmol}$ ), ethyl 3-oxopentanoate or ethyl 3-oxohexanoate or ethyl acetoacetate $(5 \mathrm{mmol})$ and piperidine $(0.5 \mathrm{mmol})$ were introduced in a $20 \mathrm{~mL}$ vial with ethanol $(10 \mathrm{~mL})$ as solution. Subsequently, the reaction vial was closed and then prestirred for $10 \mathrm{~s}$. The mixture was irradiated at $90^{\circ} \mathrm{C}$ for $10 \mathrm{~min}$. After the completion, the reaction mixture was then cooled to room temperature and concentrated in vacuo to remove the solvent. The residue was then washed with water, filtered, dried, and the precipitate was purified by recrystallization from $95 \% \mathrm{EtOH}$ to give the products of 4 . The analytical data for represent compounds are shown below. ${ }^{1} \mathrm{H}-\mathrm{NMR}$ and ${ }^{13} \mathrm{C}-\mathrm{NMR}$ spectra of compounds 4 in Supplementary Materials.

\subsubsection{2-Butyryl-3H-benzo[f]chromen-3-one (4a)}

Yellow solid; yield 89\%; m.p.: 127-129 ${ }^{\circ} \mathrm{C}$; IR (KBr): $v$ 1734, 1626, 1557, 1513, 1383, 1109, $864 \mathrm{~cm}^{-1}$; ${ }^{1} \mathrm{H}-\mathrm{NMR}\left(\mathrm{CDCl}_{3}, 400 \mathrm{MHz}\right) \delta(\mathrm{ppm}): 9.21(\mathrm{~s}, 1 \mathrm{H}, \mathrm{ArH}), 8.91(\mathrm{~s}, 1 \mathrm{H}, \mathrm{ArH}), 8.06(\mathrm{~d}, J=8.8 \mathrm{~Hz}, 1 \mathrm{H}, \mathrm{ArH})$, $7.83(\mathrm{~d}, J=8.8 \mathrm{~Hz}, 1 \mathrm{H}, \mathrm{ArH}), 7.57-7.56(\mathrm{~m}, 1 \mathrm{H}, \mathrm{ArH}), 7.20(\mathrm{~d}, J=9.2 \mathrm{~Hz}, 1 \mathrm{H}, \mathrm{ArH}), 7.12\left(\mathrm{dd}, J_{1}=8.8 \mathrm{~Hz}\right.$, $\left.J_{2}=2.0 \mathrm{~Hz}, 1 \mathrm{H}, \mathrm{ArH}\right), 3.00\left(\mathrm{t}, J=7.2 \mathrm{~Hz}, 2 \mathrm{H}, \mathrm{CH}_{2}\right), 1.63-1.58\left(\mathrm{~m}, 2 \mathrm{H}, \mathrm{CH}_{2}\right), 0.93\left(\mathrm{t}, J=7.2 \mathrm{~Hz}, 3 \mathrm{H}, \mathrm{CH}_{3}\right)$; ${ }^{13} \mathrm{C}-\mathrm{NMR}\left(100 \mathrm{MHz}, \mathrm{DMSO}-d_{6}\right) \delta$ (ppm): 197.5, 159.1, 158.9, 156.4, 142.8, 136.6, 132.1, 131.5, 124.6, 121.9, 118.6, 113.0, 111.4, 104.8, 43.9, 17.3, 14.1;

\subsubsection{2-Butyryl-9-methoxy-3H-benzo[f]chromen-3-one (4b)}

Yellow solid, yield 88\%; m.p.: $125-128^{\circ} \mathrm{C}$; IR (KBr) v: 1730, 1667, 1601, 1556, 1513, 1386, 1365, 1196, 948, $836 \mathrm{~cm}^{-1} ;{ }^{1} \mathrm{H}-\mathrm{NMR}\left(\mathrm{CDCl}_{3}, 400 \mathrm{MHz}\right) \delta(\mathrm{ppm}): 9.01(\mathrm{~s}, 1 \mathrm{H}, \mathrm{ArH}), 7.86(\mathrm{~d}, J=8.8 \mathrm{~Hz}, 1 \mathrm{H}, \mathrm{ArH}), 7.68(\mathrm{~d}$, $J=8.8 \mathrm{~Hz}, 1 \mathrm{H}, \mathrm{ArH}), 7.40(\mathrm{~s}, 1 \mathrm{H}, \mathrm{ArH}), 7.14(\mathrm{t}, J=8.4 \mathrm{~Hz}, 2 \mathrm{H}, \mathrm{ArH}), 3.94\left(\mathrm{~s}, 3 \mathrm{H}, \mathrm{CH}_{3} \mathrm{O}\right), 3.12(\mathrm{t}, J=8.4$ $\left.\mathrm{Hz}, 2 \mathrm{H}, \mathrm{CH}_{2}\right) ; 1.76-1.70\left(\mathrm{~m}, 2 \mathrm{H}, \mathrm{CH}_{2}\right), 1.00\left(\mathrm{t}, J=8.4 \mathrm{~Hz}, 3 \mathrm{H}, \mathrm{CH}_{3}\right) ;{ }^{13} \mathrm{C}-\mathrm{NMR}\left(100 \mathrm{MHz}, \mathrm{CDCl}_{3}\right) \delta$ (ppm): 197.6, 159.9, 158.7, 156.0, 142.5, 135.3, 131.2, 130.2, 124.7, 121.1, 117.9, 113.1, 111.4, 100.7, 55.5, $44.0,16.9,13.3$.

\subsubsection{2-Propionyl-3H-benzo[f]chromen-3-one (4c)}

Yellow solid, yield 87\%; m.p.: $134-136^{\circ} \mathrm{C}$; IR (KBr): $v$ 1732, 1662, 1601, 1556, 1524, 1387, 1365, 1196, 945, $823 \mathrm{~cm}^{-1} ;{ }^{1} \mathrm{H}-\mathrm{NMR}\left(\mathrm{CDCl}_{3}, 400 \mathrm{MHz}\right) \delta(\mathrm{ppm}): 9.15(\mathrm{~s}, 1 \mathrm{H}, \mathrm{ArH}), 8.74(\mathrm{~s}, 1 \mathrm{H}, \mathrm{ArH}), 7.90(\mathrm{~d}, J=8.8 \mathrm{~Hz}$, $1 \mathrm{H}, \mathrm{ArH}), 7.66(\mathrm{~d}, J=8.8 \mathrm{~Hz}, 1 \mathrm{H}, \mathrm{ArH}), 7.41-7.40(\mathrm{~m}, 1 \mathrm{H}, \mathrm{ArH}), 7.04(\mathrm{~d}, J=8.8 \mathrm{~Hz}, 1 \mathrm{H}, \mathrm{ArH}), 6.95(\mathrm{dd}$, $\left.J_{1}=8.8 \mathrm{~Hz}, J_{2}=2.0 \mathrm{~Hz}, 1 \mathrm{H}, \mathrm{ArH}\right), 3.14-3.08\left(\mathrm{~m}, 2 \mathrm{H}, \mathrm{CH}_{2}\right), 1.08\left(\mathrm{t}, J=7.2 \mathrm{~Hz}, 3 \mathrm{H}, \mathrm{CH}_{3}\right) ;{ }^{13} \mathrm{C}-\mathrm{NMR}(100$ $\left.\mathrm{MHz}, \mathrm{CDCl}_{3}\right) \delta(\mathrm{ppm}): 198.4,159.9,159.4,156.7,143.3,135.9,131.9,130.8,125.3,121.8,118.7,113.7$, $112.1,102.0,35.2,10.7$.

\subsubsection{9-Methoxy-2-propionyl-3H-benzo[f]chromen-3-one (4d)}

Yellow solid, yield 87\%; m.p.: 125-128 ${ }^{\circ} \mathrm{C}$; IR (KBr): v 1730, 1667, 1601, 1556, 1513, 1386, 1365, 1196, 948, $836 \mathrm{~cm}^{-1} ;{ }^{1} \mathrm{H}-\mathrm{NMR}$ (DMSO- $\left.d_{6}, 400 \mathrm{MHz}\right) \delta(\mathrm{ppm}): 9.19(\mathrm{~s}, 1 \mathrm{H}, \mathrm{ArH}), 8.14(\mathrm{~d}, J=9.2 \mathrm{~Hz}, 1 \mathrm{H}, \mathrm{ArH}), 7.90$ $(\mathrm{d}, J=9.2 \mathrm{~Hz}, 1 \mathrm{H}, \mathrm{ArH}), 7.79(\mathrm{~s}, 1 \mathrm{H}, \mathrm{ArH}), 7.32(\mathrm{t}, J=8.8 \mathrm{~Hz}, 1 \mathrm{H}, \mathrm{ArH}), 7.21\left(\mathrm{dd}, J_{1}=8.8 \mathrm{~Hz}, J_{2}=2.0\right.$ $\mathrm{Hz}, 1 \mathrm{H}, \mathrm{ArH}), 3.98\left(\mathrm{~s}, 3 \mathrm{H}, \mathrm{CH}_{3} \mathrm{O}\right), 3.10-3.05\left(\mathrm{~m}, 2 \mathrm{H}, \mathrm{CH}_{2}\right), 1.09\left(\mathrm{t}, J=7.2 \mathrm{~Hz}, 3 \mathrm{H}, \mathrm{CH}_{3}\right) ;{ }^{13} \mathrm{C}-\mathrm{NMR}(100$ MHz, DMSO- $\left.d_{6}\right) \delta$ (ppm): 198.7, 160.4, 158.8, 156.1, 143.0, 136.2, 131.9, 131.2, 125.4, 122.8, 118.6, 113.9, $112.1,102.4,56.2,35.4,8.4$.

\subsubsection{2-Acetyl-3H-benzo[f]chromen-3-one (4e)}

Yellow solid, yield 88\%; m.p.: 189-190 C [48]; IR (KBr): v 2959, 1696, 1622, 1562, 1384, 1227, 1206, $857 \mathrm{~cm}^{-1}$. 


\subsection{General Procedure for the Synthesis of Products $\mathbf{7}$ and $\mathbf{9}$ Are Represented as Follows}

Typically, benzo[f]chromen-3-one $4(0.5 \mathrm{mmol})$, enaminone $2(0.5 \mathrm{mmol})$, alkyl alcohol $5(1.0 \mathrm{~mL})$ or aryl alcohols $8(1.0 \mathrm{~mL})$ and SSA $(0.25 \mathrm{~g})$ were introduced in a $5 \mathrm{~mL}$ vial with ethylene glycol $(3 \mathrm{~mL})$ as solution. Subsequently, the reaction vial was closed and then prestirred for $10 \mathrm{~s}$. The mixture was irradiated at $140{ }^{\circ} \mathrm{C}$. The reaction was monitored by TLC. After the completion, the reaction mixture was then cooled to room temperature and diluted with cold water $(30 \mathrm{~mL})$, and extracted with $\mathrm{CH}_{2} \mathrm{Cl}_{2}(3 \times 30 \mathrm{~mL})$. The extracts were washed with water $(3 \times 50 \mathrm{~mL})$ and dried over anhydrous $\mathrm{Na}_{2} \mathrm{SO}_{4}$. After evaporation of the solvent under reduced pressure, the precipitate was collected and purified by recrystallization from $95 \% \mathrm{EtOH}$ or by flash column chromatography (petroleum ether:ethyl acetate $=8: 1$ ) to give the products 7 or 9 . The analytical data for represent compounds are shown below. ${ }^{1} \mathrm{H}-\mathrm{NMR}$ and ${ }^{13} \mathrm{C}-\mathrm{NMR}$ spectra of compounds 7 and 9 in Supplementary Materials.

\subsubsection{2-(5-Ethyl-3,4-dimethyl-1-phenyl-1H-pyrazolo[3,4-b]pyridin-6-yl)-3H-benzo[f]chromen-3-one (7a)}

White solid, m.p.: $258-260{ }^{\circ} \mathrm{C}$; IR $\left(\mathrm{KBr}, \mathrm{cm}^{-1}\right)$ v: 2960, 1722, 1629, 1572, 1507, 1415, 1387, 1315, 1290, 1248, 1211, 1096, 989, 906, 815, 787, 713, 691, 605; ${ }^{1} \mathrm{H}-\mathrm{NMR}\left(400 \mathrm{MHz}, \mathrm{DMSO}-d_{6}\right) \delta$ (ppm): 9.07 (s, $1 \mathrm{H}$, ArH), $8.58(\mathrm{~d}, J=8.0 \mathrm{~Hz}, 1 \mathrm{H}, \mathrm{ArH}), 8.23(\mathrm{t}, J=8.0 \mathrm{~Hz}, 3 \mathrm{H}, \mathrm{ArH}), 8.08(\mathrm{~d}, J=8.0 \mathrm{~Hz}, 1 \mathrm{H}, \operatorname{ArH}), 7.69-7.61$ $(\mathrm{m}, 3 \mathrm{H}, \mathrm{ArH}), 7.44(\mathrm{t}, J=8.0 \mathrm{~Hz}, 2 \mathrm{H}, \mathrm{ArH}), 7.20(\mathrm{t}, J=7.2 \mathrm{~Hz}, 1 \mathrm{H}, \mathrm{ArH}), 2.78-2.66\left(\mathrm{~m}, 8 \mathrm{H}, 2 \times \mathrm{CH}_{3}+\right.$ $\left.\mathrm{CH}_{2}\right), 1.05\left(\mathrm{t}, J=7.2 \mathrm{~Hz}, 3 \mathrm{H}, \mathrm{CH}_{3}\right) ;{ }^{13} \mathrm{C}-\mathrm{NMR}$ (75 MHz, $\left.\mathrm{CF}_{3} \mathrm{COOD}\right) \delta$ (ppm): 156.0, 148.8, 146.2, 145.4, 140.5, 139.1, 135.6, 134.1, 132.6, 132.0, 131.2, 130.8, 130.2, 129.4, 128.5, 126.5, 122.3, 121.3, 116.7, 116.2, 22.7, 17.0, 14.3, 13.4; HRMS: $m / z$ cacld. for $\mathrm{C}_{29} \mathrm{H}_{24} \mathrm{~N}_{3} \mathrm{O}_{2}[\mathrm{M}+\mathrm{H}]^{+} 446.1869$, Found 446.1853 .

\subsubsection{2-(4,5-Diethyl-3-methyl-1-phenyl-1H-pyrazolo[3,4-b]pyridin-6-yl)-3H-benzo[f]chromen-3-one (7b)}

White solid, m.p.: $>300{ }^{\circ} \mathrm{C}$; IR $\left(\mathrm{KBr}, \mathrm{cm}^{-1}\right)$ v: 2974, 1719, 1688, 1656, 1628, 1596, 1628, 1571, 1546, 1506, 1413, 1357, 1204, 1071, 909, 817, 752, 694, 676, 589; ${ }^{1} \mathrm{H}-\mathrm{NMR}\left(400 \mathrm{MHz}, \mathrm{DMSO}-d_{6}\right) \delta(\mathrm{ppm}): 9.16(\mathrm{~s}, 1 \mathrm{H}$, ArH), $8.66(\mathrm{~d}, J=8.4 \mathrm{~Hz}, 1 \mathrm{H}, \mathrm{ArH}), 8.29(\mathrm{~d}, J=9.2 \mathrm{~Hz}, 1 \mathrm{H}, \mathrm{ArH}), 8.21(\mathrm{~d}, J=7.6 \mathrm{~Hz}, 2 \mathrm{H}, \mathrm{ArH}), 8.11(\mathrm{~d}, J$ $=8.0 \mathrm{~Hz}, 1 \mathrm{H}, \mathrm{ArH}), 7.73-7.63(\mathrm{~m}, 3 \mathrm{H}, \mathrm{ArH}), 7.47(\mathrm{t}, J=8.0 \mathrm{~Hz}, 2 \mathrm{H}, \mathrm{ArH}), 7.23(\mathrm{t}, J=7.6 \mathrm{~Hz}, 1 \mathrm{H}, \mathrm{ArH})$, 3.51-3.48 (m, 2H, CH 2$), 3.17-3.14\left(\mathrm{~m}, 2 \mathrm{H}, \mathrm{CH}_{2}\right), 2.79\left(\mathrm{~s}, 3 \mathrm{H}, \mathrm{CH}_{3}\right), 1.35\left(\mathrm{t}, J=7.2 \mathrm{~Hz}, 3 \mathrm{H}, \mathrm{CH}_{3}\right), 1.09(\mathrm{t}$, $\left.J=7.6 \mathrm{~Hz}, 3 \mathrm{H}, \mathrm{CH}_{3}\right) ;{ }^{13} \mathrm{C}-\mathrm{NMR}\left(75 \mathrm{MHz}, \mathrm{DMSO}-d_{6}\right) \delta(\mathrm{ppm}): 160.2,154.3,153.8,149.4,148.1,142.4$, $139.6,134.1,130.5,130.3,129.6,129.0,128.3,126.8,125.7,123.2,120.5,117.2,115.9,113.5,100.0,22.2,21.6$, 16.6, 16.2, 15.5; HRMS: $m / z$ cacld. for $\mathrm{C}_{30} \mathrm{H}_{25} \mathrm{~N}_{3} \mathrm{O}_{2}(\mathrm{M})^{+} 459.1947$, Found 459.1946.

\subsubsection{2-(5-Ethyl-3-methyl-1-phenyl-4-propyl-1H-pyrazolo[3,4-b]pyridin-6-yl)-3H-benzo[f]chromen-3-one (7c)}

White solid, m.p.: $242-245^{\circ} \mathrm{C}$; IR $\left(\mathrm{KBr}, \mathrm{cm}^{-1}\right)$ v: 2974, 2880, 2703, 2545, 1789, 1722, 1665, 1573, 1503, 1439, 1414, 1389, 1359, 1320, 1288, 1248, 1217, 1155, 1091, 915, 858, 813, 792, 745, 695, 641, 610; ${ }^{1} \mathrm{H}-\mathrm{NMR}$ $\left(400 \mathrm{MHz}, \mathrm{DMSO}-d_{6}\right) \delta(\mathrm{ppm}): 9.14(\mathrm{~s}, 1 \mathrm{H}, \mathrm{ArH}), 8.63(\mathrm{~d}, J=8.8 \mathrm{~Hz}, 1 \mathrm{H}, \mathrm{ArH}), 8.26(\mathrm{~d}, J=8.8 \mathrm{~Hz}, 1 \mathrm{H}$, ArH), $8.22(\mathrm{~d}, J=8.0 \mathrm{~Hz}, 2 \mathrm{H}, \mathrm{ArH}), 8.09(\mathrm{~d}, J=8.0 \mathrm{~Hz}, 1 \mathrm{H}, \mathrm{ArH}), 7.70-7.63(\mathrm{~m}, 3 \mathrm{H}, \mathrm{ArH}), 7.46(\mathrm{t}, J$ $=7.6 \mathrm{~Hz}, 2 \mathrm{H}, \mathrm{ArH}), 7.22(\mathrm{t}, J=7.6 \mathrm{~Hz}, 1 \mathrm{H}, \mathrm{ArH}), 3.07-3.06\left(\mathrm{~m}, 2 \mathrm{H}, \mathrm{CH}_{2}\right), 2.76-2.73\left(\mathrm{~m}, 5 \mathrm{H}, \mathrm{CH}_{3}+\right.$ $\left.\mathrm{CH}_{2}\right), 1.72-1.69\left(\mathrm{~m}, 2 \mathrm{H}, \mathrm{CH}_{2}\right), 1.13\left(\mathrm{~s}, 3 \mathrm{H}, \mathrm{CH}_{3}\right), 1.07\left(\mathrm{~s}, 3 \mathrm{H}, \mathrm{CH}_{3}\right) ;{ }^{13} \mathrm{C}-\mathrm{NMR}\left(75 \mathrm{MHz}, \mathrm{CF}_{3} \mathrm{COOD}\right) \delta$ (ppm): 165.7, 155.9, 148.1, 146.2, 145.9, 140.8, 139.1, 135.0, 134.1, 132.6, 131.9, 131.2, 130.7, 130.2, 129.3, $128.5,126.5,121.6,121.3,116.3,33.4,26.2,22.2,14.5,13.7 ;$ HRMS: $m / z$ cacld. for $\mathrm{C}_{31} \mathrm{H}_{28} \mathrm{~N}_{3} \mathrm{O}_{2}[\mathrm{M}+\mathrm{H}]^{+}$ 474.2182, Found 474.2210.

\subsubsection{2-(5-Ethyl-3,4-dimethyl-1-phenyl-1H-pyrazolo[3,4-b]pyridin-6-yl)-9-methoxy-3H-benzo[f]chromen-} 3-one $(7 \mathrm{~d})$

White solid, m.p.: $>300{ }^{\circ} \mathrm{C}$; IR $\left(\mathrm{KBr}, \mathrm{cm}^{-1}\right)$ v: 2975, 2026, 1795, 1728, 1628, 1574, 1509, 1230, 1091, 989, 917, 840, 794, 751, 686, 610; ${ }^{1} \mathrm{H}-\mathrm{NMR}\left(400 \mathrm{MHz}, \mathrm{DMSO}-d_{6}\right) \delta$ (ppm): 9.21 (s, 1H, ArH), 8.24-8.17 (m, 3H, ArH), 8.01-7.96 (m, 2H, ArH), 7.50-7.45 (m, 3H, ArH), 7.27-7.21 (m, 2H, ArH), 3.90 (s, 3H, OCH $\mathrm{O}_{3}$, 2.80-2.79 (m, 8H, CH $\left.+2 \times \mathrm{CH}_{3}\right), 1.07\left(\mathrm{t}, J=7.2 \mathrm{~Hz}, 3 \mathrm{H}, \mathrm{CH}_{3}\right) ;{ }^{13} \mathrm{C}-\mathrm{NMR}\left(75 \mathrm{MHz}, \mathrm{DMSO}-d_{6}\right) \delta(\mathrm{ppm})$ : $160.3,160.1,154.4,154.0,149.0,143.1,142.4,140.0,139.7,133.8,131.4,131.0,129.5,127.5,125.6,125.5$, 
$120.3,118.7,116.9,114.3,102.6,56.3,22.4,16.1,15.4,15.0$; HRMS: $m / z$ cacld. for $\mathrm{C}_{30} \mathrm{H}_{26} \mathrm{~N}_{3} \mathrm{O}_{3}[\mathrm{M}+\mathrm{H}]^{+}$ 476.1974, Found 476.1980.

\subsubsection{2-(5-Ethyl-3-methyl-1,4-diphenyl-1H-pyrazolo[3,4-b]pyridin-6-yl)-3H-benzo[f]chromen-3-one (9a)}

Yellow solid, m.p.: $>300{ }^{\circ} \mathrm{C}$; IR $\left(\mathrm{KBr}, \mathrm{cm}^{-1}\right)$ v: 3032, 2978, 2888, 2763, 1725, 1049, 958, 815, 756, 699, 679, 588; ${ }^{1} \mathrm{H}-\mathrm{NMR}\left(400 \mathrm{MHz}, \mathrm{CF}_{3} \mathrm{COOD}\right) \delta(\mathrm{ppm}): 10.16$ (s, $\left.1 \mathrm{H}, \mathrm{ArH}\right), 9.15-9.14(\mathrm{~m}, 2 \mathrm{H}, \mathrm{ArH}), 8.88-8.87$ (m, $1 \mathrm{H}, \mathrm{ArH}), 8.65-8.64(\mathrm{~m}, 1 \mathrm{H}, \mathrm{ArH}), 8.59-8.55(\mathrm{~m}, 4 \mathrm{H}, \mathrm{ArH}), 8.51-8.47(\mathrm{~m}, 6 \mathrm{H}, \mathrm{ArH}), 8.38-8.37$ (m, 2H, ArH), 3.77-3.76 (m, 2H, CH $\mathrm{CH}_{2}, 3.05\left(\mathrm{~s}, 3 \mathrm{H}, \mathrm{CH}_{3}\right), 1.91\left(\mathrm{~s}, 3 \mathrm{H}, \mathrm{CH}_{3}\right) ;{ }^{13} \mathrm{C}-\mathrm{NMR}$ (75 MHz, $\left.\mathrm{CF}_{3} \mathrm{COOD}\right) \delta$ (ppm): 163.7, 156.1, 149.4, 146.8, 146.4, 140.8, 139.1, 135.8, 134.3, 132.8, 132.6, 132.0, 131.6, 131.2, 130.8, 130.2, 129.9, 129.5, 128.5, 127.8, 126.5, 121.9, 121.4, 116.8, 116.3, 113.6, 22.9, 14.3, 12.5; HRMS: $m / z$ cacld. for $\mathrm{C}_{34} \mathrm{H}_{26} \mathrm{~N}_{3} \mathrm{O}_{2}[\mathrm{M}+\mathrm{H}]^{+}$508.2025, Found 508.2025.

4.3.6. 2-(5-Ethyl-3-methyl-1-phenyl-4-(p-tolyl)-1H-pyrazolo[3,4-b]pyridin-6-yl)-3H-benzo[f]chromen-3one $(9 \mathbf{b})$

Yellow solid, m.p.: $>300^{\circ} \mathrm{C}$; $\mathrm{IR}\left(\mathrm{KBr}, \mathrm{cm}^{-1}\right)$ v: $2968,1972,1779,1572,1505,1413,1360,1207,1088,961$, 898, 806, 758, 728, 690, 642; ${ }^{1} \mathrm{H}-\mathrm{NMR}\left(400 \mathrm{MHz}, \mathrm{CF}_{3} \mathrm{COOD}\right) \delta$ (ppm): 10.13 (s, 1H, ArH), 9.15-9.09 (m, $2 \mathrm{H}, \mathrm{ArH}), 8.87-8.84(\mathrm{~m}, 1 \mathrm{H}, \mathrm{ArH}), 8.63-8.60(\mathrm{~m}, 1 \mathrm{H}, \mathrm{ArH}), 8.54-8.40(\mathrm{~m}, 9 \mathrm{H}, \mathrm{ArH}), 8.25-8.24(\mathrm{~m}, 2 \mathrm{H}$, $\mathrm{ArH}), 3.76-3.74\left(\mathrm{~m}, 2 \mathrm{H}, \mathrm{CH}_{2}\right), 3.37\left(\mathrm{~s}, 3 \mathrm{H}, \mathrm{CH}_{3}\right), 3.06\left(\mathrm{~s}, 3 \mathrm{H}, \mathrm{CH}_{3}\right), 1.88-1.87\left(\mathrm{~m}, 3 \mathrm{H}, \mathrm{CH}_{3}\right) ;{ }^{13} \mathrm{C}-\mathrm{NMR}$ (75 MHz, $\left.\mathrm{CF}_{3} \mathrm{COOD}\right) \delta(\mathrm{ppm}): 162.8,155.1,148.6,145.6,145.4,142.0,139.8,138.2,135.0,133.3,131.7$, $131.1,130.3,129.9,129.6,129.3,128.7,128.5,127.6,126.9,125.6,121.0,120.4,115.9,115.4,112.7,21.9,19.5$, 13.4, 11.7; HRMS: $m / z$ cacld. for $\mathrm{C}_{35} \mathrm{H}_{28} \mathrm{~N}_{3} \mathrm{O}_{2}[\mathrm{M}+\mathrm{H}]^{+}$522.2182, Found 522.2180.

4.3.7. 2-(5-Ethyl-4-(4-methoxyphenyl)-3-methyl-1-phenyl-1H-pyrazolo[3,4-b]pyridin-6-yl)-3H-benzo[f] chromen-3-one $(9 \mathrm{c})$

Yellow solid, m.p.: $>300^{\circ} \mathrm{C}$; IR $\left(\mathrm{KBr}, \mathrm{cm}^{-1}\right)$ v: 2967, 1711, 1597, 1571, 1505, 1412, 1286, 1249, 1211, $1048,982,897,849,806,758,690,641,587 ;{ }^{1} \mathrm{H}-\mathrm{NMR}\left(400 \mathrm{MHz}, \mathrm{CF}_{3} \mathrm{COOD}\right) \delta$ (ppm): 9.29 (s, 1H, ArH), 8.28-8.23 (m, 2H, ArH), $7.99(\mathrm{~d}, J=8.4 \mathrm{~Hz}, 1 \mathrm{H}, \mathrm{ArH}), 7.76(\mathrm{t}, J=7.6 \mathrm{~Hz}, 1 \mathrm{H}, \mathrm{ArH}), 7.69-7.56(\mathrm{~m}, 7 \mathrm{H}$, $\mathrm{ArH}), 7.50(\mathrm{~d}, J=8.4 \mathrm{~Hz}, 2 \mathrm{H}, \mathrm{ArH}), 7.35(\mathrm{~d}, J=8.4 \mathrm{~Hz}, 2 \mathrm{H}, \mathrm{ArH}), 4.06\left(\mathrm{~s}, 3 \mathrm{H}, \mathrm{OCH}_{3}\right), 2.93-2.88(\mathrm{~m}, 2 \mathrm{H}$, $\left.\mathrm{CH}_{2}\right), 2.23\left(\mathrm{~s}, 3 \mathrm{H}, \mathrm{CH}_{3}\right), 1.02\left(\mathrm{t}, J=7.2 \mathrm{~Hz}, 3 \mathrm{H}, \mathrm{CH}_{3}\right) ;{ }^{13} \mathrm{C}-\mathrm{NMR}\left(75 \mathrm{MHz}, \mathrm{CF}_{3} \mathrm{COOD}\right) \delta(\mathrm{ppm}): 162.9$, $160.9,160.5,155.2,148.4,145.8,145.7,140.0,138.3,135.3,133.5,131.8,131.2,130.4,130.0,129.4,129.2$, $128.7,127.7,125.8,125.3,121.3,120.6,116.0,115.5,114.9,112.8,55.1,22.1,13.4,12.0$; HRMS: $\mathrm{m} / z$ cacld. for $\mathrm{C}_{35} \mathrm{H}_{28} \mathrm{~N}_{3} \mathrm{O}_{3}[\mathrm{M}+\mathrm{H}]^{+}$538.2131, Found 538.2111.

4.3.8. 2-(5-Ethyl-4-(3-methoxyphenyl)-3-methyl-1-phenyl-1H-pyrazolo[3,4-b]pyridin-6-yl)-3H-benzo[f] chromen-3-one (9d)

Yellow solid, m.p.: $>300{ }^{\circ} \mathrm{C}$; IR $\left(\mathrm{KBr}, \mathrm{cm}^{-1}\right)$ v: 2965, 2023, 1785, 1712, 1573, 1504, 1382, 1357, 1285, 1158, 1136, 1046, 782, 759, 712, 689, 588; ${ }^{1} \mathrm{H}-\mathrm{NMR}\left(400 \mathrm{MHz}, \mathrm{DMSO}-d_{6}\right) \delta(\mathrm{ppm}): 9.23$ (s, $\left.1 \mathrm{H}, \mathrm{ArH}\right), 8.66(\mathrm{~d}$, $J=8.4 \mathrm{~Hz}, 1 \mathrm{H}, \mathrm{ArH}), 8.29-8.23(\mathrm{~m}, 3 \mathrm{H}, \mathrm{ArH}), 8.10(\mathrm{~d}, J=8.0 \mathrm{~Hz}, 1 \mathrm{H}, \mathrm{ArH}), 7.73-7.62(\mathrm{~m}, 3 \mathrm{H}, \mathrm{ArH})$, 7.54-7.47 (m, 3H, ArH), $7.25(\mathrm{t}, J=7.6 \mathrm{~Hz}, 1 \mathrm{H}, \mathrm{ArH}), 7.14-7.12(\mathrm{~m}, 1 \mathrm{H}, \mathrm{ArH}), 7.03-7.01(\mathrm{~m}, 2 \mathrm{H}, \mathrm{ArH})$, $3.84\left(\mathrm{~s}, 3 \mathrm{H}, \mathrm{OCH}_{3}\right), 2.58-2.56\left(\mathrm{~m}, 2 \mathrm{H}, \mathrm{CH}_{2}\right), 1.96\left(\mathrm{~s}, 3 \mathrm{H}, \mathrm{CH}_{3}\right), 0.89\left(\mathrm{t}, J=7.2 \mathrm{~Hz}, 3 \mathrm{H}, \mathrm{CH}_{3}\right) ;{ }^{13} \mathrm{C}-\mathrm{NMR}$ (75 MHz, DMSO- $d_{6}$ ) $\delta$ (ppm): 160.2, 159.6, 154.2, 153.9, 148.7, 145.3, 142.9, 140.1, 139.6, 137.3, 134.3, 130.5, 130.4, 130.1, 129.6, 129.5, 129.4, 129.0, 127.8, 126.8, 125.9, 123.1, 121.4, 120.6, 117.2, 115.8, 114.6, 113.5, 55.8, 22.5, 16.0, 14.2; HRMS: $m / z$ cacld. for $\mathrm{C}_{35} \mathrm{H}_{27} \mathrm{~N}_{3} \mathrm{O}_{3}(\mathrm{M})^{+}$537.2052, Found 537.2053.

4.3.9. 2-(4-(4-Bromophenyl)-5-ethyl-3-methyl-1-phenyl-1H-pyrazolo[3,4-b]pyridin-6-yl)-3H-benzo[f] chromen-3-one $(9 \mathbf{9})$

Yellow solid, m.p.: $>300^{\circ} \mathrm{C}$;.IR $\left(\mathrm{KBr}, \mathrm{cm}^{-1}\right)$ v: 2968, 2032, 1775, 1721, 1574, 1385, 1357, 1285, 1166, 1047, 782, 759, 712, 681, 588; ${ }^{1} \mathrm{H}-\mathrm{NMR}\left(400 \mathrm{MHz}, \mathrm{DMSO}-d_{6}\right) \delta(\mathrm{ppm}): 10.19(\mathrm{~s}, 1 \mathrm{H}, \mathrm{ArH}), 8.57-8.53(\mathrm{~m}, 2 \mathrm{H}$, ArH), $8.43(\mathrm{~d}, J=9.2 \mathrm{~Hz}, 1 \mathrm{H}, \mathrm{ArH}), 8.06(\mathrm{~d}, J=8.0 \mathrm{~Hz}, 1 \mathrm{H}, \mathrm{ArH}), 7.86(\mathrm{t}, J=7.6 \mathrm{~Hz}, 1 \mathrm{H}, \mathrm{ArH}), 7.78-7.23$ $(\mathrm{m}, 10 \mathrm{H}, \mathrm{ArH}), 2.79\left(\mathrm{~s}, 2 \mathrm{H}, \mathrm{CH}_{2}\right), 2.54\left(\mathrm{~s}, 3 \mathrm{H}, \mathrm{CH}_{3}\right), 1.35\left(\mathrm{t}, J=7.2 \mathrm{~Hz}, 3 \mathrm{H}, \mathrm{CH}_{3}\right) ;{ }^{13} \mathrm{C}-\mathrm{NMR}(75 \mathrm{MHz}$, 
DMSO- $\left.d_{6}\right) \delta$ (ppm): 165.7, 159.3, 157.7, 152.3, 151.4, 144.7, 141.6, 138.9, 134.8, 132.3, 131.0, 129.7, 126.7, 125.2, 121.4, 118.1, 113.1, 111.4, 111.3, 109.6, 107.5, 21.9, 17.0, 14.6; HRMS: $m / z$ cacld. for $\mathrm{C}_{34} \mathrm{H}_{24} \mathrm{BrN}_{3} \mathrm{O}_{2}$ $(\mathrm{M})^{+}$585.1052, Found 585.1057.

4.3.10. 2-(5-Ethyl-3-methyl-1-phenyl-4-(pyridin-4-yl)-1H-pyrazolo[3,4-b]pyridin-6-yl)-3H-benzo[f] chromen-3-one (9f):

Yellow solid, m.p.: $>300{ }^{\circ} \mathrm{C}$; IR $\left(\mathrm{KBr}, \mathrm{cm}^{-1}\right)$ v: 2965, 1972, 1783, 1573, 1505, 1413, 1362, 1089, 961, 898, 805, 758, 693, 642; ${ }^{1} \mathrm{H}-\mathrm{NMR}$ (400 MHz, $\left.\mathrm{CF}_{3} \mathrm{COOD}\right) \delta$ (ppm): 10.09 (s, 1H, ArH), 9.11-9.05 (m, 2H, ArH), $8.80(\mathrm{~d}, J=8.0 \mathrm{~Hz}, 1 \mathrm{H}, \mathrm{ArH}), 8.60-8.35(\mathrm{~m}, 10 \mathrm{H}, \mathrm{ArH}), 8.21-8.19(\mathrm{~m}, 2 \mathrm{H}, \mathrm{ArH}), 3.72-3.70\left(\mathrm{~m}, 2 \mathrm{H}, \mathrm{CH}_{2}\right)$, $3.01\left(\mathrm{~s}, 3 \mathrm{H}, \mathrm{CH}_{3}\right), 1.83\left(\mathrm{t}, J=6.8 \mathrm{~Hz}, 3 \mathrm{H}, \mathrm{CH}_{3}\right) ;{ }^{13} \mathrm{C}-\mathrm{NMR}\left(75 \mathrm{MHz}, \mathrm{CF}_{3} \mathrm{COOD}\right) \delta(\mathrm{ppm}): 162.8,155.0$, 148.5, 145.5, 145.4, 142.0, 139.7, 138.1, 135.0, 133.3, 131.6, 131.0, 130.2, 129.8, 129.5, 129.2, 128.7, 128.5, 127.5, 126.8, 125.5, 121.0, 120.4, 115.8, 115.3, 112.6, 21.8, 13.3, 11.6; HRMS: $m / z$ cacld. for $\mathrm{C}_{33} \mathrm{H}_{25} \mathrm{~N}_{4} \mathrm{O}_{2}$ $[\mathrm{M}+\mathrm{H}]^{+}$509.1978, Found 509.1963.

4.3.11. 2-(5-Ethyl-4-(furan-2-yl)-3-methyl-1-phenyl-1H-pyrazolo[3,4-b]pyridin-6-yl)-3H-benzo[f]chromen3-one (9g):

Yellow solid, m.p.: $>300^{\circ} \mathrm{C}$; IR $\left(\mathrm{KBr}, \mathrm{cm}^{-1}\right)$ v: 2966, 1720, 1629, 1566, 1412, 1383, 1264, 1084, 959, 852, 797, 766, 724, 691, 640, 617; ${ }^{1} \mathrm{H}-\mathrm{NMR}\left(400 \mathrm{MHz}, \mathrm{CF}_{3} \mathrm{COOD}\right) \delta$ (ppm): 9.24 (s, 1H, ArH), 8.21 (d, J = 9.2 $\mathrm{Hz}, 1 \mathrm{H}, \mathrm{ArH}), 7.95(\mathrm{~d}, J=8.8 \mathrm{~Hz}, 1 \mathrm{H}, \mathrm{ArH}), 7.65-7.63(\mathrm{~m}, 2 \mathrm{H}, \mathrm{ArH}), 7.62-7.55(\mathrm{~m}, 6 \mathrm{H}, \mathrm{ArH}), 7.46(\mathrm{~d}, J$ $=9.2 \mathrm{~Hz}, 1 \mathrm{H}, \mathrm{ArH}), 7.39(\mathrm{~m}, 3 \mathrm{H}, \mathrm{ArH}), 2.93-2.87\left(\mathrm{~m}, 2 \mathrm{H}, \mathrm{CH}_{2}\right), 2.21\left(\mathrm{~s}, 3 \mathrm{H}, \mathrm{CH}_{3}\right), 1.03(\mathrm{t}, J=7.6 \mathrm{~Hz}$, $\left.3 \mathrm{H}, \mathrm{CH}_{3}\right) ;{ }^{13} \mathrm{C}-\mathrm{NMR}\left(75 \mathrm{MHz}, \mathrm{CF}_{3} \mathrm{COOD}\right) \delta(\mathrm{ppm}): 163.0,160.2,156.0,148.7,145.7,142.1,140.0,137.9$, 135.2, 133.5, 131.9, 131.5, 130.7, 130.5, 129.8, 129.0, 127.1, 126.9, 125.8, 121.2, 118.0, 115.4, 113.7, 112.2, 22.1, 13.6, 11.8; HRMS: $m / z$ cacld. for $\mathrm{C}_{32} \mathrm{H}_{24} \mathrm{~N}_{3} \mathrm{O}_{3}[\mathrm{M}+\mathrm{H}]^{+} 498.1818$, Found 498.1831.

4.3.12. 2-(5-Ethyl-3-methyl-1,4-diphenyl-1H-pyrazolo[3,4-b]pyridin-6-yl)-9-methoxy-3H-benzo[f]chromen3-one (9h)

White solid, m.p.: $248-250^{\circ} \mathrm{C}$; IR $\left(\mathrm{KBr}, \mathrm{cm}^{-1}\right)$ v: 2968, 1724, 1631, 1573, 1507, 1434, 1414, 1384, 1354, 1281, 1241, 1135, 1105, 980, 960, 905, 827, 789, 758, 705, 692, 636; ${ }^{1} \mathrm{H}-\mathrm{NMR}\left(400 \mathrm{MHz}\right.$, DMSO-d $\left.d_{6}\right) \delta$ (ppm): $9.32(\mathrm{~s}, 1 \mathrm{H}, \mathrm{ArH}), 8.24(\mathrm{~d}, J=8.0 \mathrm{~Hz}, 2 \mathrm{H}, \mathrm{ArH}), 8.17(\mathrm{~d}, J=9.2 \mathrm{~Hz}, 1 \mathrm{H}, \mathrm{ArH}), 8.00-7.97(\mathrm{~m}, 2 \mathrm{H}, \mathrm{ArH})$, 7.61-7.57 (m, 3H, ArH), 7.51-7.46 (m, 5H, ArH), 7.27-7.24 (m, 2H, ArH), 3.91 (s, 3H, OCH $\left.\mathrm{OCH}_{3}\right), 2.54-2.53$ $\left(\mathrm{m}, 2 \mathrm{H}, \mathrm{CH}_{2}\right), 1.89\left(\mathrm{~s}, 3 \mathrm{H}, \mathrm{CH}_{3}\right), 0.86\left(\mathrm{t}, J=7.6 \mathrm{~Hz}, 3 \mathrm{H}, \mathrm{CH}_{3}\right) ;{ }^{13} \mathrm{C}-\mathrm{NMR}\left(75 \mathrm{MHz}, \mathrm{DMSO}-d_{6}\right) \delta(\mathrm{ppm}):$ 160.3 , 160.2, 154.5, 154.4, 148.7, 145.5, 142.8, 140.6, 139.5, 135.9, 134.0, 131.5, 131.0, 130.5, 129.6, 129.1, 129.0, 128.9, 127.0, 125.9, 125.7, 120.6, 118.7, 115.8, 114.3, 112.8, 102.7, 56.3, 22.5, 15.8, 14.2; HRMS: $\mathrm{m} / \mathrm{z}$ cacld. for $\mathrm{C}_{35} \mathrm{H}_{28} \mathrm{~N}_{3} \mathrm{O}_{3}[\mathrm{M}+\mathrm{H}]^{+}$538.2131, Found 538.2122.

4.3.13. 2-(5-Ethyl-3-methyl-1-phenyl-4-(p-tolyl)-1H-pyrazolo[3,4-b]pyridin-6-yl)-9-methoxy-3H-benzo[f] chromen-3-one (9i)

Yellow solid, m.p.: $>300{ }^{\circ} \mathrm{C}$; IR $\left(\mathrm{KBr}, \mathrm{cm}^{-1}\right)$ v: 2966, 1720, 1628, 1570, 1417, 1383, 1264, 1084, 959, 904, 832, 796, 761, 725, 691, 678, 640, 602; ${ }^{1} \mathrm{H}-\mathrm{NMR}$ (400 MHz, $\left.\mathrm{CF}_{3} \mathrm{COOD}\right) \delta$ (ppm): 9.24 (s, 1H, ArH), 8.20 $(\mathrm{d}, J=9.2 \mathrm{~Hz}, 1 \mathrm{H}, \mathrm{ArH}), 7.95(\mathrm{~d}, J=8.8 \mathrm{~Hz}, 1 \mathrm{H}, \mathrm{ArH}), 7.65-7.54(\mathrm{~m}, 8 \mathrm{H}, \operatorname{ArH}), 7.46(\mathrm{~d}, J=9.2 \mathrm{~Hz}, 1 \mathrm{H}$, $\mathrm{ArH}), 7.39(\mathrm{~d}, J=7.6 \mathrm{~Hz}, 3 \mathrm{H}, \mathrm{ArH}), 4.04\left(\mathrm{~s}, 3 \mathrm{H}, \mathrm{OCH}_{3}\right), 2.92-2.87\left(\mathrm{~m}, 2 \mathrm{H}, \mathrm{CH}_{2}\right), 2.52\left(\mathrm{~s}, 3 \mathrm{H}, \mathrm{CH}_{3}\right), 2.20$ $\left(\mathrm{s}, 3 \mathrm{H}, \mathrm{CH}_{3}\right), 1.02\left(\mathrm{t}, J=7.6 \mathrm{~Hz}, 3 \mathrm{H}, \mathrm{CH}_{3}\right) ;{ }^{13} \mathrm{C}-\mathrm{NMR}\left(75 \mathrm{MHz}, \mathrm{CF}_{3} \mathrm{COOD}\right) \delta(\mathrm{ppm}): 162.9,160.1,155.9$, 148.6, 145.6, 145.5, 142.0, 139.8, 137.8, 135.1, 133.4, 131.8, 131.4, 130.6, 130.4, 129.6, 128.9, 127.0, 126.8, 125.7, 121.1, 117.9, 115.3, 113.6, 112.1, 55.3, 22.0, 19.6, 13.5, 11.8; HRMS: $m / z$ cacld. for $\mathrm{C}_{36} \mathrm{H}_{30} \mathrm{~N}_{3} \mathrm{O}_{3}[\mathrm{M}+$ $\mathrm{H}]^{+}$552.2287, Found 552.2246.

4.3.14. 2-(5-Ethyl-4-(4-methoxyphenyl)-3-methyl-1-phenyl-1H-pyrazolo[3,4-b]pyridin-6-yl)-9-methoxy3H-benzolffchromen-3-one $\mathbf{( 9 \mathbf { j } )}$

White solid, m.p.: $256-258^{\circ} \mathrm{C}$; IR $\left(\mathrm{KBr}, \mathrm{cm}^{-1}\right)$ v: 2965, 2145, 1735, 1717, 1629, 1572, 1463, 1381, 1286, 1227, 1077, 960, 887, 884, 805, 691, 604, 567; ${ }^{1} \mathrm{H}-\mathrm{NMR}$ (400 MHz, DMSO-d $\left.d_{6}\right) \delta$ (ppm): 9.33 (s, $\left.1 \mathrm{H}, \mathrm{ArH}\right)$, 
$8.23(\mathrm{~d}, J=8.0 \mathrm{~Hz}, 2 \mathrm{H}, \mathrm{ArH}), 8.19(\mathrm{~d}, J=8.8 \mathrm{~Hz}, 1 \mathrm{H}, \mathrm{ArH}), 8.01-7.99(\mathrm{~m}, 2 \mathrm{H}, \mathrm{ArH}), 7.52-7.48(\mathrm{~m}, 3 \mathrm{H}$, $\mathrm{ArH}), 7.39-7.38(\mathrm{~m}, 2 \mathrm{H}, \mathrm{ArH}), 7.28-7.25(\mathrm{~m}, 2 \mathrm{H}, \mathrm{ArH}), 7.16(\mathrm{~d}, J=8.8 \mathrm{~Hz}, 2 \mathrm{H}, \mathrm{ArH}), 3.92\left(\mathrm{~s}, 3 \mathrm{H}, \mathrm{OCH}_{3}\right)$, $2.87\left(\mathrm{~s}, 3 \mathrm{H}, \mathrm{OCH}_{3}\right), 2.56-2.55\left(\mathrm{~m}, 2 \mathrm{H}, \mathrm{CH}_{2}\right), 1.95\left(\mathrm{~s}, 3 \mathrm{H}, \mathrm{CH}_{3}\right), 0.87\left(\mathrm{t}, J=7.2 \mathrm{~Hz}, 3 \mathrm{H}, \mathrm{CH}_{3}\right) ;{ }^{13} \mathrm{C}-\mathrm{NMR}$ (75 MHz, DMSO- $\left.d_{6}\right) \delta$ (ppm): 160.3, 160.2, 159.7, 154.6, 154.4, 148.8, 145.5, 142.9, 140.6, 139.6, 134.0, 131.5, 131.0, 130.9, 130.4, 129.7, 127.8, 127.1, 125.9, 125.7, 120.6, 118.8, 116.2, 114.3, 112.8, 102.7, 56.4, 55.7, 22.5, 15.8, 14.5; HRMS: $m / z$ cacld. for $\mathrm{C}_{36} \mathrm{H}_{30} \mathrm{~N}_{3} \mathrm{O}_{4}[\mathrm{M}+\mathrm{H}]^{+}$568.2236, Found 568.2248.

\subsubsection{2-(3,5-Dimethyl-1,4-diphenyl-1H-pyrazolo[3,4-b]pyridin-6-yl)-3H-benzo[f]chromen-3-one (9k)}

Yellow solid, m.p.: $>300^{\circ} \mathrm{C}$; IR $\left(\mathrm{KBr}, \mathrm{cm}^{-1}\right)$ v: 2934, 2173, 1710, 1598, 1572, 1438, 1278, 965, 909, 820, 791, 692, 651, 633, 585; ${ }^{1} \mathrm{H}-\mathrm{NMR}$ (400 MHz, CF 3 COOD) $\delta$ (ppm): 9.33 (s, 1H, ArH), 8.29-8.24 (m, 2H, ArH), 8.00-7.97 (m, 1H, ArH), 7.72-7.61 (m, 11H, ArH), 7.48-7.47 (m, 2H, ArH), $2.42\left(\mathrm{~s}, 3 \mathrm{H}, \mathrm{CH}_{3}\right), 2.22(\mathrm{~s}$, $\left.3 \mathrm{H}, \mathrm{CH}_{3}\right) ;{ }^{13} \mathrm{C}-\mathrm{NMR}\left(75 \mathrm{MHz}, \mathrm{CF}_{3} \mathrm{COOD}\right) \delta$ (ppm): 155.3, 148.3, 146.2, 145.8, 139.9, 138.5, 133.5, 132.4, 131.8, 131.1, 130.8, 130.4, 130.0, 129.4, 129.3, 129.0, 128.7, 127.7, 126.9, 125.7, 120.6, 120.4, 115.9, 115.5, 112.9, 14.5, 12.0; HRMS: $m / z$ cacld. for $\mathrm{C}_{33} \mathrm{H}_{24} \mathrm{~N}_{3} \mathrm{O}_{2}[\mathrm{M}+\mathrm{H}]^{+} 494.1869$, Found 494.1887.

\subsubsection{2-(3,5-Dimethyl-1-phenyl-4-(p-tolyl)-1H-pyrazolo[3,4-b]pyridin-6-yl)-3H-benzo[f]chromen-3-one (91)}

Yellow solid, m.p.: $286-290^{\circ} \mathrm{C}$; IR (KBr, cm $\left.{ }^{-1}\right)$ v: 3078, 2187, 1719, 1626, 1606, 1575, 1507, 1447, 1380, 1212, 1093, 963, 813, 790, 741, 685; ${ }^{1} \mathrm{H}-\mathrm{NMR}\left(400 \mathrm{MHz}, \mathrm{CF}_{3} \mathrm{COOD}\right) \delta$ (ppm): 9.35 (s, 1H, ArH), 8.31 (d, J $=9.2 \mathrm{~Hz}, 1 \mathrm{H}, \mathrm{ArH}), 8.27(\mathrm{~d}, J=7.6 \mathrm{~Hz}, 1 \mathrm{H}, \mathrm{ArH}), 8.02(\mathrm{~d}, J=8.4 \mathrm{~Hz}, 1 \mathrm{H}, \mathrm{ArH}), 7.79(\mathrm{t}, J=7.2 \mathrm{~Hz}, 1 \mathrm{H}$, $\mathrm{ArH}), 7.72-7.57(\mathrm{~m}, 9 \mathrm{H}, \mathrm{ArH}), 7.39(\mathrm{~d}, J=7.6 \mathrm{~Hz}, 2 \mathrm{H}, \mathrm{ArH}), 2.55\left(\mathrm{~s}, 3 \mathrm{H}, \mathrm{CH}_{3}\right), 2.46\left(\mathrm{~s}, 3 \mathrm{H}, \mathrm{CH}_{3}\right), 2.29(\mathrm{~s}$, $\left.3 \mathrm{H}, \mathrm{CH}_{3}\right) ;{ }^{13} \mathrm{C}-\mathrm{NMR}\left(75 \mathrm{MHz}, \mathrm{CF}_{3} \mathrm{COOD}\right) \delta$ (ppm): 154.3, 147.5, 145.2, 144.5, 141.3, 138.9, 137.5, 132.5, $130.8,130.2,129.4,129.0,128.8,128.4,128.3,128.1,127.7,126.7,126.0,124.7,119.6,119.5,114.9,114.4$, 18.6, 14.0, 10.9; HRMS: $m / z$ cacld. for $\mathrm{C}_{34} \mathrm{H}_{26} \mathrm{~N}_{3} \mathrm{O}_{2}[\mathrm{M}+\mathrm{H}]^{+}$508.2025, Found 508.2020.

4.3.17. 2-(4-(4-Methoxyphenyl)-3,5-dimethyl-1-phenyl-1H-pyrazolo[3,4-b]pyridin-6-yl)-3H-benzo [f]chromen-3-one $(9 \mathrm{~m})$

White solid, m.p.: $258-260^{\circ} \mathrm{C}$; IR $\left(\mathrm{KBr}, \mathrm{cm}^{-1}\right)$ v: 2904, 2342, 1735, 1631, 1574, 1427, 1367, 1240, 1200 , $1158,1103,1061,849,818,759,712,668,589 ;{ }^{1} \mathrm{H}-\mathrm{NMR}\left(400 \mathrm{MHz}, \mathrm{DMSO}-d_{6}\right) \delta(\mathrm{ppm}): 9.20(\mathrm{~s}, 1 \mathrm{H}, \mathrm{ArH})$, $8.66(\mathrm{~d}, J=8.4 \mathrm{~Hz}, 1 \mathrm{H}, \mathrm{ArH}), 8.28(\mathrm{t}, J=7.6 \mathrm{~Hz}, 3 \mathrm{H}, \mathrm{ArH}), 8.11(\mathrm{~d}, J=8.0 \mathrm{~Hz}, 1 \mathrm{H}, \mathrm{ArH}), 7.75-7.63(\mathrm{~m}$, $3 \mathrm{H}, \mathrm{ArH}), 7.50(\mathrm{t}, J=8.0 \mathrm{~Hz}, 2 \mathrm{H}, \mathrm{ArH}), 7.37(\mathrm{~d}, J=8.4 \mathrm{~Hz}, 2 \mathrm{H}, \mathrm{ArH}), 7.26(\mathrm{t}, J=7.2 \mathrm{~Hz}, 1 \mathrm{H}, \mathrm{ArH}), 7.16$ $(\mathrm{d}, J=8.4 \mathrm{~Hz}, 2 \mathrm{H}, \mathrm{ArH}), 3.87\left(\mathrm{~s}, 3 \mathrm{H}, \mathrm{OCH}_{3}\right), 2.13\left(\mathrm{~s}, 3 \mathrm{H}, \mathrm{CH}_{3}\right), 2.02\left(\mathrm{~s}, 3 \mathrm{H}, \mathrm{CH}_{3}\right) ;{ }^{13} \mathrm{C}-\mathrm{NMR}(75 \mathrm{MHz}$, DMSO- $\left.d_{6}\right) \delta$ (ppm): 159.8, 159.6, 154.2, 154.0, 149.0, 145.3, 142.7, 140.3, 139.6, 134.3, 130.6, 130.5, 129.6 129.5, 128.1, 126.7, 125.8, 124.9, 123.1, 120.6, 117.1, 115.9, 114.4, 113.6, 55.7, 16.3, 14.7; HRMS: $m / z$ cacld. for $\mathrm{C}_{34} \mathrm{H}_{26} \mathrm{~N}_{3} \mathrm{O}_{3}[\mathrm{M}+\mathrm{H}]^{+}$524.1974, Found 524.1978.

\subsubsection{2-(4-(3-Methoxyphenyl)-3,5-dimethyl-1-phenyl-1H-pyrazolo[3,4-b]pyridin-6-yl)-3H-benzo[f]} chromen-3-one (9n)

White solid, m.p.: $260-263{ }^{\circ} \mathrm{C}$; IR $\left(\mathrm{KBr}, \mathrm{cm}^{-1}\right)$ v: 2970, 2372, 1718, 1573, 1505, 1410, 1362, 1279, 1239, $1142,1054,1019,988,970,877,815,786,744,714,670,586 ;{ }^{1} \mathrm{H}-\mathrm{NMR}\left(400 \mathrm{MHz}, \mathrm{DMSO}-d_{6}\right) \delta$ (ppm): $9.19(\mathrm{~s}, 1 \mathrm{H}, \mathrm{ArH}), 8.66(\mathrm{~d}, J=8.4 \mathrm{~Hz}, 1 \mathrm{H}, \mathrm{ArH}), 8.29-8.25(\mathrm{~m}, 3 \mathrm{H}, \mathrm{ArH}), 8.10(\mathrm{~d}, J=8.0 \mathrm{~Hz}, 1 \mathrm{H}, \mathrm{ArH})$, 7.75-7.63 (m, 3H, ArH), 7.52-7.48 (m, 3H, ArH), 7.25 (t, $J=7.6 \mathrm{~Hz}, 1 \mathrm{H}, \mathrm{ArH}), 7.13-7.11(\mathrm{~m}, 1 \mathrm{H}, \mathrm{ArH})$, 7.00-6.98 (m, 2H, ArH), $3.84\left(\mathrm{~s}, 3 \mathrm{H}, \mathrm{OCH}_{3}\right), 2.13\left(\mathrm{~s}, 3 \mathrm{H}, \mathrm{CH}_{3}\right), 2.00\left(\mathrm{~s}, 3 \mathrm{H}, \mathrm{CH}_{3}\right) ;{ }^{13} \mathrm{C}-\mathrm{NMR}(75 \mathrm{MHz}$, DMSO- $\left.d_{6}\right) \delta(\mathrm{ppm}): 159.7,159.6,154.3,154.0,148.9,145.2,142.7,140.4,139.6,137.6,134.3,130.5,130.3$, 129.6, 129.5, 129.4, 129.0, 128.1, 126.8, 125.9, 124.5, 123.1, 121.3, 120.6, 117.1, 115.5, 114.7, 114.6, 113.6, 55.8, 16.2, 14.4; HRMS: $m / z$ cacld. for $\mathrm{C}_{34} \mathrm{H}_{26} \mathrm{~N}_{3} \mathrm{O}_{3}[\mathrm{M}+\mathrm{H}]^{+}$524.1974, Found 524.1978.

4.3.19. 2-(3,5-Dimethyl-1-phenyl-4-(p-tolyl)-1H-pyrazolo[3,4-b]pyridin-6-yl)-9-methoxy-3H-benzo[f] chromen-3-one (9o)

Yellow solid, m.p.: $288-290^{\circ} \mathrm{C}$; IR $\left(\mathrm{KBr}, \mathrm{cm}^{-1}\right)$ v: 2929, 1718, 1631, 1600, 1346, 1239, 1204, 1173, 1149 , $1125,1019,852,827,795,749,690,643,606 ;{ }^{1} \mathrm{H}-\mathrm{NMR}$ (400 MHz, CF 3 COOD) $\delta$ (ppm): 9.28 (s, $\left.1 \mathrm{H}, \mathrm{ArH}\right)$, 
$8.22(\mathrm{~d}, J=8.8 \mathrm{~Hz}, 1 \mathrm{H}, \mathrm{ArH}), 7.96(\mathrm{~d}, J=8.8 \mathrm{~Hz}, 1 \mathrm{H}, \mathrm{ArH}), 7.65-7.62(\mathrm{~m}, 6 \mathrm{H}, \mathrm{ArH}), 7.56-7.54(\mathrm{~m}, 2 \mathrm{H}$, $\mathrm{ArH}), 7.46(\mathrm{~d}, J=8.8 \mathrm{~Hz}, 1 \mathrm{H}, \mathrm{ArH}), 7.41-7.35(\mathrm{~m}, 3 \mathrm{H}, \mathrm{ArH}), 4.04\left(\mathrm{~s}, 3 \mathrm{H}, \mathrm{OCH}_{3}\right), 2.52\left(\mathrm{~s}, 3 \mathrm{H}, \mathrm{CH}_{3}\right), 2.44$

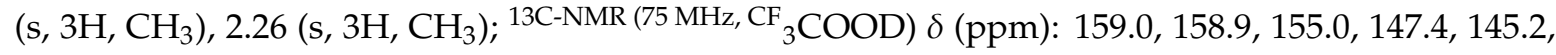
144.4, 141.2, 138.8, 137.0, 132.4, 130.7, 130.4, 129.6, 129.4, 128.8, 128.2, 128.1, 126.0, 125.8, 124.6, 119.4, 116.6, 115.2, 114.1, 112.5, 54.2, 18.5, 14.0, 10.9; HRMS: $m / z$ cacld. for $\mathrm{C}_{35} \mathrm{H}_{28} \mathrm{~N}_{3} \mathrm{O}_{3}[\mathrm{M}+\mathrm{H}]^{+} 538.2131$, Found 538.2130.

4.3.20. 9-Methoxy-2-(4-(4-methoxyphenyl)-3,5-dimethyl-1-phenyl-1H-pyrazolo[3,4-b]pyridin-6-yl)-3Hbenzo[f]chromen-3-one $(\mathbf{9 p})$

Yellow solid, m.p.: 287-289 ${ }^{\circ} \mathrm{C}$; IR $\left(\mathrm{KBr}, \mathrm{cm}^{-1}\right)$ v: 1716, 1630, 1611, 1571, 1513, 1464, 1385, 1246, 1107, 1033, 960, 832, 795, 754, 691; ${ }^{1} \mathrm{H}-\mathrm{NMR}\left(400 \mathrm{MHz}, \mathrm{CF}_{3} \mathrm{COOD}\right) \delta(\mathrm{ppm}): 9.29$ (s, $\left.1 \mathrm{H}, \mathrm{ArH}\right), 8.23$ (d, $J=8.8$ $\mathrm{Hz}, 1 \mathrm{H}, \mathrm{ArH}), 7.56(\mathrm{~d}, J=8.8 \mathrm{~Hz}, 1 \mathrm{H}, \mathrm{ArH}), 7.65-7.63(\mathrm{~m}, 6 \mathrm{H}, \mathrm{ArH}), 7.48-7.42(\mathrm{~m}, 3 \mathrm{H}, \mathrm{ArH}), 7.39-7.35(\mathrm{~m}$,

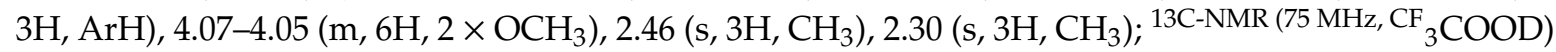
$\delta$ (ppm): 159.6, 159.0, 155.0, 147.2, 145.1, 144.6, 138.9, 137.0, 132.4, 130.7, 130.4, 129.6, 129.3, 128.3, 128.1, 125.8, 124.6, 116.6, 114.0, 112.5, 54.3, 54.0, 14.0, 11.1; HRMS: $m / z$ cacld. for $\mathrm{C}_{35} \mathrm{H}_{28} \mathrm{~N}_{3} \mathrm{O}_{4}[\mathrm{M}+\mathrm{H}]^{+}$ 554.2080, Found 554.2093.

4.3.21. 2-(3,5-Dimethyl-1,4-diphenyl-1H-pyrazolo[3,4-b]pyridin-6-yl)-9-methoxy-3H-benzo[f]chromen-3one $(\mathbf{9 q})$

Yellow solid, m.p.: $252-254{ }^{\circ} \mathrm{C}$; IR $\left(\mathrm{KBr}, \mathrm{cm}^{-1}\right)$ v: 2961, 1725, 1629, 1582, 1557, 1435, 1397, 1335, 1290, 1250, 1219, 1196, 999, 906, 819, 797, 753, 695, 625; ${ }^{1} \mathrm{H}-\mathrm{NMR}\left(400 \mathrm{MHz}, \mathrm{DMSO}-d_{6}\right) \delta(\mathrm{ppm}): 9.29$ (s, $1 \mathrm{H}$, ArH), 8.28-8.26 (m, 2H, ArH), $8.17(\mathrm{~d}, J=9.2 \mathrm{~Hz}, 1 \mathrm{H}, \mathrm{ArH}), 8.01-7.98(\mathrm{~m}, 2 \mathrm{H}, \mathrm{ArH}), 7.62-7.57(\mathrm{~m}, 3 \mathrm{H}$, $\mathrm{ArH}), 7.52-7.43(\mathrm{~m}, 5 \mathrm{H}, \mathrm{ArH}), 7.28-7.25(\mathrm{~m}, 2 \mathrm{H}, \mathrm{ArH}), 3.92\left(\mathrm{~s}, 3 \mathrm{H}, \mathrm{OCH}_{3}\right), 2.11\left(\mathrm{~s}, 3 \mathrm{H}, \mathrm{CH}_{3}\right), 1.95(\mathrm{~s}, 3 \mathrm{H}$, $\left.\mathrm{CH}_{3}\right) ;{ }^{13} \mathrm{C}-\mathrm{NMR}\left(75 \mathrm{MHz}\right.$, DMSO- $\left.d_{6}\right) \delta$ (ppm): 160.2, 159.7, 154.6, 154.5, 148.9, 145.3, 142.6, 140.8, 139.6, 136.2, 134.0, 131.5, 131.1, 129.7, 129.2, 129.1, 127.4, 125.9, 125.7, 124.5, 120.7, 118.7, 115.5, 114.3, 112.9, 102.8, 56.3, 16.2, 14.5; HRMS: $m / z$ cacld. for $\mathrm{C}_{34} \mathrm{H}_{26} \mathrm{~N}_{3} \mathrm{O}_{3}[\mathrm{M}+\mathrm{H}]^{+}$524.1974, Found 524.1988.

\subsubsection{2-(3-Methyl-1,4-diphenyl-1H-pyrazolo[3,4-b]pyridin-6-yl)-3H-benzo[f]chromen-3-one (9r)}

Yellow solid, m.p.: $268-270{ }^{\circ} \mathrm{C}$; IR $\left(\mathrm{KBr}_{\mathrm{cm}}{ }^{-1}\right)$ v: 2935, 2355, 1729, 1667, 1553, 1092, 891, 818, 746, 694, 657, 631, 585; ${ }^{1} \mathrm{H}-\mathrm{NMR}\left(400 \mathrm{MHz}, \mathrm{CF}_{3} \mathrm{COOD}\right) \delta$ (ppm): 10.22 (s, 1H, ArH), 8.61-8.57 (m, 2H, ArH), 8.46 $(\mathrm{d}, J=9.2 \mathrm{~Hz}, 1 \mathrm{H}, \mathrm{ArH}), 8.09(\mathrm{~d}, J=8.0 \mathrm{~Hz}, 1 \mathrm{H}, \mathrm{ArH}), 7.90(\mathrm{t}, J=7.6 \mathrm{~Hz}, 1 \mathrm{H}, \mathrm{ArH}), 7.82-7.76(\mathrm{~m}, 11 \mathrm{H}$, $\mathrm{ArH}), 7.70(\mathrm{~d}, J=8.8 \mathrm{~Hz}, 1 \mathrm{H}, \mathrm{ArH}), 2.58\left(\mathrm{~s}, 3 \mathrm{H}, \mathrm{CH}_{3}\right) ;{ }^{13 \mathrm{C}-\mathrm{NMR}}\left(75 \mathrm{MHz}, \mathrm{CF}_{3} \mathrm{COOD}\right) \delta(\mathrm{ppm}): 163.5,159.1$, 155.0, 147.5, 145.0, 144.8, 140.3, 138.7, 132.9, 132.4, 131.1, 130.4, 130.3, 130.0, 129.8, 128.8, 128.3, 127.9, 127.4, 127.3, 122.6, 120.1, 114.3, 114.1, 113.5, 11.9; HRMS: $m / z$ cacld. for $\mathrm{C}_{32} \mathrm{H}_{22} \mathrm{~N}_{3} \mathrm{O}_{2}[\mathrm{M}+\mathrm{H}]^{+} 480.1712$, Found 480.1726 .

4.3.23. 2-(4-(4-Methoxyphenyl)-3-methyl-1-phenyl-1H-pyrazolo[3,4-b]pyridin-6-yl)-3H-benzo[f]chromen-3one $(9 \mathrm{~s})$

Yellow solid, m.p.: $>300^{\circ} \mathrm{C}$; $\mathrm{IR}\left(\mathrm{KBr}, \mathrm{cm}^{-1}\right)$ v: 2988, 2355, 1987, 1730, 1512, 1089, 1066, 959, 810, 809, 788, 765, 689, 654, 633, 599; ${ }^{1} \mathrm{H}-\mathrm{NMR}\left(400 \mathrm{MHz}, \mathrm{CF}_{3} \mathrm{COOD}\right) \delta(\mathrm{ppm}): 11.03$ (s, $\left.1 \mathrm{H}, \mathrm{ArH}\right), 9.43$ (d, J = $8.4 \mathrm{~Hz}$, 1H, ArH), 9.34-9.30 (m, 2H, ArH), $8.94(\mathrm{~d}, J=8.0 \mathrm{~Hz}, 1 \mathrm{H}, \mathrm{ArH}), 8.75(\mathrm{t}, J=7.6 \mathrm{~Hz}, 1 \mathrm{H}, \mathrm{ArH}), 7.66-7.63$ $(\mathrm{m}, 8 \mathrm{H}, \mathrm{ArH}), 8.54(\mathrm{t}, J=9.2 \mathrm{~Hz}, 1 \mathrm{H}, \mathrm{ArH}), 8.23(\mathrm{~d}, J=8.8 \mathrm{~Hz}, 2 \mathrm{H}, \mathrm{ArH}), 4.95\left(\mathrm{~s}, 3 \mathrm{H}, \mathrm{OCH}_{3}\right), 3.50(\mathrm{~s}, 3 \mathrm{H}$, $\left.\mathrm{CH}_{3}\right)$; $13 \mathrm{C}-\mathrm{NMR}\left(75 \mathrm{MHz}, \mathrm{CF}_{3} \mathrm{COOD}\right) \delta$ (ppm): $164.3,159.2,155.9,148.2,145.7,145.3,141.1,139.8,133.6$, 131.3, 130.9, 130.6, 129.7, 128.8, 128.2, 126.6, 123.5, 120.9, 117.1, 115.1, 114.6, 55.1, 13.1; HRMS: $m / z$ cacld. for $\mathrm{C}_{33} \mathrm{H}_{24} \mathrm{~N}_{3} \mathrm{O}_{3}[\mathrm{M}+\mathrm{H}]^{+}$510.1818, Found 510.1835.

4.3.24. 2-(5-Ethyl-1-methyl-3,4-diphenyl-1H-pyrazolo[3,4-b]pyridin-6-yl)-3H-benzo[f]chromen-3-one (9t)

Yellow solid, m.p.: $285-288^{\circ} \mathrm{C}$; IR $\left(\mathrm{KBr}, \mathrm{cm}^{-1}\right)$ v: 2396, 1732, 1574, 1353, 1099, 1515, 1088, 1076, 959, 810, 803, 704; ${ }^{1} \mathrm{H}-\mathrm{NMR}\left(400 \mathrm{MHz}, \mathrm{CF}_{3} \mathrm{COOD}\right) \delta(\mathrm{ppm}): 10.26$ (s, $\left.1 \mathrm{H}, \mathrm{ArH}\right), 9.25$ (d, J = 8.8 Hz, 2H, $\operatorname{ArH}), 8.95(\mathrm{~d}, J=8.0 \mathrm{~Hz}, 1 \mathrm{H}, \mathrm{ArH}), 8.73(\mathrm{t}, J=7.6 \mathrm{~Hz}, 1 \mathrm{H}, \mathrm{ArH}), 8.63(\mathrm{t}, J=7.6 \mathrm{~Hz}, 1 \mathrm{H}, \operatorname{ArH}), 8.46(\mathrm{~d}$, 
$J=7.6 \mathrm{~Hz}, 1 \mathrm{H}, \mathrm{ArH}), 8.26(\mathrm{t}, J=7.6 \mathrm{~Hz}, 1 \mathrm{H}, \mathrm{ArH}), 8.21-8.14(\mathrm{~m}, 3 \mathrm{H}, \mathrm{ArH}), 8.09-8.02$ (m, 4H, ArH), $7.94(\mathrm{~d}, J=7.6 \mathrm{~Hz}, 2 \mathrm{H}, \mathrm{ArH}), 5.31\left(\mathrm{~s}, 3 \mathrm{H}, \mathrm{CH}_{3}\right), 3.87-3.85\left(\mathrm{~m}, 2 \mathrm{H}, \mathrm{CH}_{2}\right), 1.84\left(\mathrm{t}, J=7.2 \mathrm{~Hz}, 3 \mathrm{H}, \mathrm{CH}_{3}\right)$; 13C-NMR (75 MHz, $\left.\mathrm{CF}_{3} \mathrm{COOD}\right) \delta$ (ppm): 162.9, 155.3, 150.3, 146.0, 145.4, 140.3, 138.2, 134.3, 131.2, 131.1, $130.2,129.9,129.5,129.3,128.5,128.3,127.9,127.8,127.6,127.1,120.5,119.3,116.3,115.5,34.9,21.8,13.1$; HRMS: $m / z$ cacld. for $\mathrm{C}_{34} \mathrm{H}_{26} \mathrm{~N}_{3} \mathrm{O}_{2}[\mathrm{M}+\mathrm{H}]^{+}$508.2025, Found 508.2027.

4.3.25. 2-(1,5-Dimethyl-3,4-diphenyl-1H-pyrazolo[3,4-b]pyridin-6-yl)-9-methoxy-3H-benzo[f]chromen-3one $(9 \mathbf{u})$

Yellow solid, m.p.: $260-262{ }^{\circ} \mathrm{C}$; IR $\left(\mathrm{KBr}, \mathrm{cm}^{-1}\right) v: 2697,2551,1783,1708,1628,1567,1511,1469,1441$, 1387, 1330, 1218, 1149, 1017, 976, 898, 845, 796, 756, 725, 702, 601; ${ }^{1} \mathrm{H}-\mathrm{NMR}\left(400 \mathrm{MHz}, \mathrm{CF}_{3} \mathrm{COOD}\right) \delta$ (ppm): 10.10 (s, 1H, ArH), 9.03 (d, J = 9.2 Hz, 1H, ArH), 8.76 (d, J = 8.8 Hz, 1H, ArH), 8.66-8.65 (m, 1H, ArH), $8.28(\mathrm{~d}, J=8.8 \mathrm{~Hz}, 1 \mathrm{H}, \mathrm{ArH}), 8.20(\mathrm{~d}, J=9.2 \mathrm{~Hz}, 1 \mathrm{H}, \mathrm{ArH}), 8.12$ (d, J = 7.6 Hz, 1H, ArH), 8.05-7.99 (m, 3H, ArH), 7.91-7.87 (m, 4H, ArH), 7.81 (d, J = 7.6 Hz, 2H, ArH), $5.18\left(\mathrm{~s}, 3 \mathrm{H}, \mathrm{OCH}_{3}\right), 4.85$ $\left(\mathrm{s}, 3 \mathrm{H}, \mathrm{CH}_{3}\right), 3.21$ (s, 3H, $\left.\mathrm{CH}_{3}\right)$. ${ }^{13 \mathrm{C}-\mathrm{NMR}}\left(75 \mathrm{MHz}, \mathrm{CF}_{3} \mathrm{COOD}\right) \delta(\mathrm{ppm}): 162.3,160.0,156.0,150.1,146.1$, 145.8, 140.3, 138.0, 131.5, 131.4, 130.6, 130.3, 129.5, 128.5, 128.3, 128.2, 128.0, 127.9, 127.2, 126.7, 117.5, 113.5, 112.2, 112.1, 103.0, 55.2, 34.8, 15.1. HRMS: $m / z$ cacld. for $\mathrm{C}_{34} \mathrm{H}_{26} \mathrm{~N}_{3} \mathrm{O}_{3}[\mathrm{M}+\mathrm{H}]^{+}$554.2080, Found 554.2093.

4.3.26. 9-Methoxy-2-(4-(4-methoxyphenyl)-1,5-dimethyl-3-phenyl-1H-pyrazolo[3,4-b]pyridin-6-yl)-3Hbenzo[f]chromen-3-one $(9 \mathbf{v})$

Yellow solid, m.p.: 240-244 ${ }^{\circ} \mathrm{C}$; IR $\left(\mathrm{KBr}, \mathrm{cm}^{-1}\right)$ v: 2932, 1720, 1624, 1608, 1564, 1512, 1463, 1383, 1353, $1289,1249,1208,1173,1025,970,902,836,801,698,664,607 ;{ }^{1} \mathrm{H}-\mathrm{NMR}\left(400 \mathrm{MHz}, \mathrm{CF}_{3} \mathrm{COOD}\right) \delta(\mathrm{ppm})$ : 10.15 (s, 1H, ArH), 9.09 (d, J = 9.2 Hz, 1H, ArH), 8.82 (d, J = 9.2 Hz, 1H, ArH), 8.72-8.71 (m, 1H, ArH), $8.34(\mathrm{~d}, J=9.2 \mathrm{~Hz}, 1 \mathrm{H}, \mathrm{ArH}), 8.26(\mathrm{~d}, J=9.2 \mathrm{~Hz}, 1 \mathrm{H}, \mathrm{ArH}), 8.17-8.13(\mathrm{~m}, 1 \mathrm{H}, \mathrm{ArH}), 8.02-7.95(\mathrm{~m}, 4 \mathrm{H}$, $\mathrm{ArH}), 7.89-7.87$ (m, 2H, ArH), 7.69-7.66 (m, 2H, ArH), $5.23\left(\mathrm{~s}, 3 \mathrm{H}, \mathrm{OCH}_{3}\right), 4.90\left(\mathrm{~s}, 3 \mathrm{H}, \mathrm{OCH}_{3}\right), 4.72(\mathrm{~s}$, $\left.3 \mathrm{H}, \mathrm{CH}_{3}\right), 3.31$ (s, 3H, $\left.\mathrm{CH}_{3}\right)$; $13 \mathrm{C}-\mathrm{NMR}\left(75 \mathrm{MHz}, \mathrm{CF}_{3} \mathrm{COOD}\right) \delta(\mathrm{ppm}): 162.4,160.3,156.1,150.0,146.0,145.9$, $140.3,138.1,131.5,130.4,129.4,128.7,128.1,128.0,127.6,126.8,125.1,117.5,114.2,113.5,55.2,55.1,34.9$, 15.1; HRMS: $m / z$ cacld. for $\mathrm{C}_{35} \mathrm{H}_{28} \mathrm{~N}_{3} \mathrm{O}_{4}[\mathrm{M}+\mathrm{H}]^{+}$524.1974, Found 524.1972.

Supplementary Materials: The following are available online, Crystal date of compound 7a [47], ${ }^{1} \mathrm{H}$ NMR and ${ }^{13} \mathrm{C}$ NMR Spectra of all compounds and GC-MS spectra of Scheme 4B.

Author Contributions: W.L. conceived the synthetic route, supervised the project and wrote the paper; J.Z. and J.W. designed the experiments; C.Z. and X.H. performed all synthetic work in the laboratory.

Funding: This research was supported financially by the Natural Science Foundation of China (no. 21502074), Qing Lan Project of Jiangsu Province, Postgraduate Research \& Practice Innovation Program of Jiangsu Province (SJCX18_0985 and SJCX19_0766) and CAMS lnitiative for Innovative Medicine (2016-I2M-3-014).

Conflicts of Interest: The authors declare no conflict of interest.

\section{References and Note}

1. Revankar, H.M.; Bukhari, S.N.A.; Kumar, G.B.; Qin, H.L. Coumarins scaffolds as COX inhibitors. Bioorg. Chem. 2017, 71, 146-159. [CrossRef] [PubMed]

2. Dandriyal, J.; Singla, R.; Kumar, M.; Jaitak, V. Recent developments of C-4 substituted coumarin derivatives as anticancer agents. Eur. J. Med. Chem. 2016, 119, 141-168. [CrossRef] [PubMed]

3. Ibrar, A.; Shehzadi, S.A.; Saeed, F.; Khan, I. Developing hybrid molecule therapeutics for diverse enzyme inhibitory action: Active role of coumarin-based structural leads in drug discovery. Bioorg. Med. Chem. 2018, 26, 3731-3762. [CrossRef] [PubMed]

4. Singla, S.; Piplani, P. Coumarin derivatives as potential inhibitors of acetylcholinesterase: Synthesis, molecular docking and biological studies. Bioorg. Med. Chem. 2016, 24, 4587-4599. [CrossRef] [PubMed]

5. Hamulakova, S.; Janovec, L.; Soukup, O.; Jun, D.; Kuca, K. Synthesis, in vitro acetylcholinesterase inhibitory activity and molecular docking of new acridine-coumarin hybrids. Int. J. Biol. Macromol. 2017, 104, 333-338. [CrossRef] 
6. Lan, J.S.; Ding, Y.; Liu, Y.; Kang, P.; Hou, J.W.; Zhang, X.Y.; Xie, S.S.; Zhang, T. Design, synthesis and biological evaluation of novel coumarin- $N$-benzyl pyridinium hybrids as multi-target agents for the treatment of Alzheimer's disease. Eur. J. Med. Chem. 2017, 139, 48-59. [CrossRef] [PubMed]

7. Emami, S.; Dadashpour, S. Current developments of coumarin-based anti-cancer agents in medicinal chemistry. Eur. J. Med. Chem. 2015, 102, 611-630. [CrossRef]

8. Kaur, M.; Kohli, S.; Sandhu, S.; Bansal, Y.; Bansal, G. Coumarin: A promising scaffold for anticancer agents. Anti Cancer Agents Med. Chem. 2015, 15, 1032-1048. [CrossRef]

9. Klenkar, J.; Molnar, M. Natural and synthetic coumarins as potential anticancer agents. J. Chem. Pharm. Res. 2015, 7, 1223-1238.

10. Poole, S.K.; Poole, C.F. Thin-layer chromatographic method for the determination of the principal polar aromatic flavour compounds of the cinnamons of commerce. Analyst 1994, 119, 113-120. [CrossRef]

11. Riveiro, M.E.; De Kimpe, N.; Moglioni, A.; Vazquez, R.; Monczor, F.; Shayo, C.; Davio, C. Coumarins: Old compounds with novel promising therapeutic perspectives. Curr. Med. Chem. 2010, 17, 1325-1338. [CrossRef] [PubMed]

12. Patil, A.D.; Freyer, A.J.; Eggleston, D.S.; Haltiwanger, R.C.; Bean, M.F.; Taylor, P.B.; Caranfa, M.J.; Breen, A.L.; Bartus, H.R. The inophyllums, novel inhibitors of HIV-1 reverse transcriptase isolated from the malaysian tree, calophyllum inophyllum linn. J. Med. Chem. 1993, 36, 4131-4138. [CrossRef] [PubMed]

13. Spino, C.; Dodier, M.; Sotheeswaran, S. Anti-HIV coumarins from calophyllum seed oil. Bioorg. Med. Chem. Lett. 1998, 8, 3475-3478. [CrossRef]

14. Kostova, I.; Mojzis, J. Biologically active coumarins as inhibitors of HIV-1. Future HIV Ther. 2007, 1, 315-329. [CrossRef]

15. Shin, E.; Choi, K.M.; Yoo, H.S.; Lee, C.K.; Hwang, B.Y.; Lee, M.K. Inhibitory effects of coumarins from the stem barks of Fraxinus rhynchophylla on adipocyte differentiation in 3T3-L1 cells. Biol. Pharm. Bull. 2010, 33, 1610-1614. [CrossRef] [PubMed]

16. Keri, R.S.; Sasidhar, B.S.; Nagaraja, B.M.; Santos, M.A. Recent progress in the drug development of coumarin derivatives as potent antituberculosis agents. Eur. J. Med. Chem. 2015, 100, 257-269. [CrossRef] [PubMed]

17. Piller, N.B. A comparison of the effectiveness of some anti-inflammatory drugs on thermal oedema. Br. J. Exp. Pathol. 1975, 56, 554-560. [PubMed]

18. Bansal, Y.; Sethi, P.; Bansal, G. Coumarin: A potential nucleus for anti-inflammatory molecules. Med. Chem. Res. 2013, 22, 3049-3060. [CrossRef]

19. Whang, W.K.; Park, H.S.; Ham, I.; Oh, M.; Namkoong, H.; Kim, H.K.; Hwang, D.W.; Hur, S.Y.; Kim, T.E.; Park, Y.G. Natural compounds, fraxin and chemicals structurally related to fraxin protect cells from oxidative stress. Exp. Mol. Med. 2005, 37, 436-446. [CrossRef]

20. Rosselli, S.; Maggio, A.M.; Faraone, N.; Spadaro, V.; Morris-Natschke, S.L.; Bastow, K.F.; Lee, K.H.; Bruno, M. The cytotoxic properties of natural coumarins isolated from roots of ferulago campestris (Apiaceae) and of synthetic ester derivatives of aegelinol. Nat. Prod. Commun. 2009, 4, 1701-1706. [CrossRef]

21. Crichton, E.G.; Waterman, P.G. Dihydromammea C/OB: A new coumarin from the seed of mammea Africana. Phytochemistry 1978, 17, 1783-1786. [CrossRef]

22. Baek, N.I.; Ahn, E.M.; Kim, H.Y.; Park, Y.D. Furanocoumarins from the root of Angelica dahurica. Arch. Pharm. Res. 2000, 23, 467-470. [CrossRef] [PubMed]

23. Teng, M.C.; Lin, H.; Ko, F.N.; Wu, T.S.; Huang, T.F. The relaxant action of osthole isolated from Angelica pubescens in guinea-pig trachea. Naunyn Schmiedeberg's. Arch. Pharmacol. 1994, 349, 202-208. [CrossRef] [PubMed]

24. Fort, D.; Rao, K.; Jolad, S.; Luo, J.; Carlson, T.; King, S. Antihyperglycemic activity of teramnus labialis (fabaceae). Phytomedicine 2000, 6, 465-467. [CrossRef]

25. Liu, Y.H.; Xu, J.R.; Wang, Q.; Li, M. Coupling coumarin to gold nanoparticles by DNA chains for sensitive detection of DNase I. Anal. Biochem. 2018, 555, 50-54. [CrossRef] [PubMed]

26. Chesterman, J.P.; Hughes, T.C.; Amsden, B.G. Reversibly photo-crosslinkable aliphatic polycarbonates functionalized with coumarin. Eur. Polym. J. 2018, 105, 186-193. [CrossRef]

27. Sarkar, N.; Datta, A.; Das, S.; Bhattacharyya, K. Solvation dynamics of coumarin 480 in micelles. J. Phys. Chem. 1996, 100, 15483-15486. [CrossRef]

28. Arzhantsev, S.; Ito, N.; Heitz, M.; Maroncelli, M. Solvation dynamics of coumarin 153 in several classes of ionic liquids: Cation dependence of the ultrafast component. Chem. Phys. Lett. 2003, 381, 278-286. [CrossRef] 
29. Lang, B.; Angulo, G.; Vauthey, E. Ultrafast solvation dynamics of coumarin 153 in imidazolium-based ionic liquids. J. Phys. Chem. A 2006, 110, 7028-7034. [CrossRef]

30. Birau, M.M.; Wang, Z.Y. A dual-mode molecular switch based on a chiral binaphthol-coumarin compound. Tetrahedron Lett. 2000, 41, 4025-4028. [CrossRef]

31. Deng, G.W.; Xu, H.J.; Kuang, L.; He, C.C.; Li, B.K.; Yang, M.; Zhang, X.L.; Li, Z.H.; Liu, J.L. Novel nonlinear optical chromophores based on coumarin: Synthesis and properties studies. Opt. Mater. 2019, 88, 218-222. [CrossRef]

32. Goda, F.E.; Abdel-Azizb, A.A.M.; Attef, O.A. Synthesis, antimicrobial activity and conformational analysis of novel substituted pyridines: $\mathrm{BF}_{3}$-promoted reaction of hydrazine with 2-alkoxy pyridines. Bioorg. Med. Chem. 2004, 12, 1845-1852. [CrossRef] [PubMed]

33. Foks, H.; Pancechowska-Ksepko, D.; Kędzia, A.; Zwolska, Z.; Janowiec, M.; Augustinowicz-Kopeć, E. Synthesis and antibacterial activity of $1 H$-pyrazolo[3,4-b] pyrazine and-pyridine derivatives. Farmaco 2005, 60, 513-517. [CrossRef] [PubMed]

34. Bharate, S.B.; Mahajan, T.R.; Gole, Y.R.; Nambiar, M.; Matan, T.T.; Kulkarni-Almeida, A.; Balachandran, S.; Junjappa, H.; Balakrishnan, A.; Vishwakarma, R.A. Synthesis and evaluation of pyrazolo[3,4-b] pyridines and its structural analogues as TNF- $\alpha$ and IL-6 inhibitors. Bioorg. Med. Chem. 2008, 16, 7167-7176. [CrossRef] [PubMed]

35. De Mello, H.; Echevarria, A.; Bernardino, A.M.; CantoCavalheiro, M.; Leon, L.L. Antileishmanial pyrazolopyridine derivatives: Synthesis and structure-activty relationship analysis. J. Med. Chem. 2004, 47, 5427-5432. [CrossRef] [PubMed]

36. Misra, R.N.; Rawlins, D.B.; Xiao, H.; Shan, W.; Bursuker, I.; Kellar, K.A.; Mulheron, J.G.; Sack, J.S.; Tokarski, J.S.; Kimball, S.D.; et al. 1H-Pyrazolo[3,4-b] pyridine inhibitors of cyclin-dependent kinases: Highly potent 2,6-difluorophenacyl analogues. Bioorg. Med. Chem. Lett. 2003, 13, 2405-2408. [CrossRef]

37. Lin, R.; Connolly, P.J.; Lu, Y.; Chiu, G.; Li, S.; Yu, Y.; Huang, S.; Li, X.; Emanuel, S.L.; Middleton, S.A.; et al. Synthesis and evaluation of pyrazolo[3,4-b] pyridine CDK1 inhibitors as anti-tumor agents. Bioorg. Med. Chem. Lett. 2007, 17, 4297-4302. [CrossRef] [PubMed]

38. Parker, W.B. Enzymology of purine and pyrimidine antimetabolites used in the treatment of cancer. Chem. Rev. 2009, 109, 2880-2893. [CrossRef]

39. Miliutina, M.; Janke, J.; Hassan, S.; Zaib, S.; Iqbal, J.; Lecka, J.; Sévigny, J.; Villinger, A.; Friedrich, A.; Lochbrunner, S.; et al. A domino reaction of 3-chlorochromones with aminoheterocycles. Synthesis of pyrazolopyridines and benzofuropyridines and their optical and ecto-5'-nucleotidase inhibitory effects. Org. Biomol. Chem. 2018, 16, 717-732. [CrossRef]

40. Lin, W.; Hu, X.X.; Song, S.; Cai, Q.; Wang, Y.; Shi, D.Q. Microwave-assisted synthesis of novel hetero[5] helicene-like molecules and coumarin derivatives. Org. Biomol. Chem. 2017, 15, 7909-7916. [CrossRef]

41. Wang, H.Y.; Liu, X.C.; Feng, X.; Huang, Z.B.; Shi, D.Q. GAP chemistry for pyrrolyl coumarin derivatives: A highly efficient one-pot synthesis under catalyst-free conditions. Green Chem. 2013, 15, 3307-3311. [CrossRef]

42. Wang, H.Y.; Shi, D.Q. Efficient synthesis of functionalized dihydro- $1 H$-indol-4(5H)-ones via one-pot three-component reaction under catalyst-free conditions. ACS Comb. Sci. 2013, 15, 261-266. [CrossRef] [PubMed]

43. Liu, X.C.; Lin, W.; Wang, H.Y.; Huang, Z.B.; Shi, D.Q. Improved and efficient synthesis of chromeno [4,3-d]pyrazolo[3,4- $b]$ pyridine-6(3H)-ones and their fluorescence properties. J. Heterocycl. Chem. 2014, 51, 1036-1044. [CrossRef]

44. Wang, J.X.; Lin, W.; Liu, H.T.; Hu, M.H.; Feng, X.; Ren, J.F.; Huang, Z.B.; Shi, D.Q. An efficient synthesis of coumarino[4,3- $d$ ] pyrazolo[3,4- $b]$-pyridine derivatives catalyzed by silica sulfuric acid under microwave irradiation. Chin. J. Org. Chem. 2015, 35, 927-933. [CrossRef]

45. Lin, W.; Cai, Q.; Zheng, C.Z.; Zheng, Y.X.; Shi, D.Q. Synthesis of functionalized coumarino[4,3-d]pyrazolo [3,4-b]pyridine derivatives and their selective recognition for $\mathrm{Zn}^{2+}$. Chin. J. Org. Chem. 2017, 37, 2392-2398. [CrossRef]

46. Lin, W.; Zheng, Y.X.; Wang, Y.Z.; Shi, D.Q. An efficient synthesis of functionalized chromeno[4,3- $d]$ pyrazolo [3,4-b]pyridine derivatives. Heterocycles 2016, 92, 2235-2243. 
47. Crystallographic Data for 7a Have been Deposited at the Cambridge Crystallographic Data Centre (CCDC 1881623). Available online: www.ccdc.cam.ac.uk/conts/retrieving.html (accessed on 30 July 2019).

48. Bogdal, D. Coumarins: Fast synthesis by knoevenagel condensation under microwave irradiation. J. Chem. Res. Synop. 1998, 12, 468-469. [CrossRef]

Sample Availability: Samples of the compounds 4 and 7 are available from the authors.

(C) 2019 by the authors. Licensee MDPI, Basel, Switzerland. This article is an open access article distributed under the terms and conditions of the Creative Commons Attribution (CC BY) license (http://creativecommons.org/licenses/by/4.0/). 